\title{
An Active Set Smoothing Method for Solving Unconstrained Minimax Problems
}

\author{
Zhengyong Zhou iD and Qi Yang \\ School of Mathematics and Computer Sciences, Shanxi Normal University, Linfen, Shanxi 041004, China \\ Correspondence should be addressed to Zhengyong Zhou; zzy198300@163.com
}

Received 14 February 2020; Accepted 5 June 2020; Published 24 June 2020

Academic Editor: Mohammad D. Aliyu

Copyright (c) 2020 Zhengyong Zhou and Qi Yang. This is an open access article distributed under the Creative Commons Attribution License, which permits unrestricted use, distribution, and reproduction in any medium, provided the original work is properly cited.

\begin{abstract}
In this paper, an active set smoothing function based on the plus function is constructed for the maximum function. The active set strategy used in the smoothing function reduces the number of gradients and Hessians evaluations of the component functions in the optimization. Combing the active set smoothing function, a simple adjustment rule for the smoothing parameters, and an unconstrained minimization method, an active set smoothing method is proposed for solving unconstrained minimax problems. The active set smoothing function is continuously differentiable, and its gradient is locally Lipschitz continuous and strongly semismooth. Under the boundedness assumption on the level set of the objective function, the convergence of the proposed method is established. Numerical experiments show that the proposed method is feasible and efficient, particularly for the minimax problems with very many component functions.
\end{abstract}

\section{Introduction}

In this paper, we consider the following unconstrained minimax problem:

$$
\min _{x \in R^{n}}\left\{F(x)=\max _{j \in Q} f_{j}(x)\right\},
$$

where the component functions $f_{i}: R^{n} \longrightarrow R, j \in Q=$ $\{1, \ldots, q\}$, are twice continuously differentiable. Minimax problem (1) is a typical nonsmooth optimization problem and arises in many fields, such as engineering design ([1]), vehicle routing $([2,3])$, structural optimization ([4]), electronic circuit design ([5]), and game theory $([6,7])$.

Many methods have been proposed for solving minimax problem (1), such as subgradient methods ([8]), bundle type methods $([9,10])$, cutting plane methods $([11])$, sequential quadratic programming methods ([12-14]), interior point methods ([15-17]), conjugate gradient methods ([18]), and smoothing methods ([19-26]). The main advantage of smoothing methods is that the minimax problem is transformed into a sequence of simple, smooth, and unconstrained optimization problems, which can be solved by standard unconstrained minimization solvers.
In [27], the following aggregate function (also called the exponential penalty function) induced from Jaynes' maximum entropy principle was introduced:

$$
F_{t}(x)=t \ln \left(\sum_{j \in Q} \exp \left(\frac{f_{j}(x)}{t}\right)\right),
$$

where $t>0$ is the smoothing parameter. It approaches $F(x)$ uniformly with respect to $x \in R^{n}$ as the smoothing parameter goes to 0 , and has been wildly used in the smoothing methods for solving the minimax problems. Its gradient can be written as follows:

$$
\nabla F_{t}(x)=\sum_{j \in Q} \mu_{t}^{j}(x) \nabla f_{j}(x),
$$

with

$$
\begin{aligned}
\mu_{t}^{j}(x) & =\frac{\exp \left(f_{j}(x) / t\right)}{\sum_{j \in Q} \exp \left(f_{j}(x) / t\right)} \in(0,1], \\
\sum_{j \in Q} \mu_{t}^{j}(x) & =1,
\end{aligned}
$$


which is a convex combination of the gradients of all the component functions, and its Hessian

$$
\begin{aligned}
\nabla^{2} F_{t}(x)= & \sum_{j \in Q} \mu_{t}^{j}(x) \nabla^{2} f_{j}(x)+\frac{1}{t} \sum_{j \in Q} \mu_{t}^{j}(x) \nabla f_{j}(x) \nabla f_{j}(x)^{T} \\
& -\frac{1}{t} \sum_{j \in Q} \mu_{t}^{j}(x) \nabla f_{j}(x) \sum_{j \in Q} \mu_{t}^{j}(x) \nabla f_{j}(x)^{T},
\end{aligned}
$$

is a complicated combination of the gradients and Hessians of all component functions. Therefore, for the maximum function with very many nonlinear component functions, the evaluations for the gradient and Hessian of the aggregate function always consume a large amount of computation.

For the minimax problems with very many component functions, several active set strategies have been developed for the smoothing methods to reduce the number of gradients or Hessians evaluations of the component functions at each iteration. In [18], the following active set smoothing function for $F(x, \alpha)=\alpha+\sum_{j \in Q} \max \left(f_{j}(x)-\alpha, 0\right)$ was presented:

$$
F_{t}(x, \alpha)=\alpha+\sum_{j \in Q} \varphi\left(f_{j}(x)-\alpha ; t\right)
$$

where $t>0$ is the smoothing parameter,

$$
\varphi(z ; t)= \begin{cases}0, & z<-t, \\ \frac{(z+t)^{3}}{6 t^{2}}, & -t \leq z \leq 0, \\ z+\frac{(z-t)^{3}}{6 t^{2}}, & 0<z \leq t, \\ z, & z>t .\end{cases}
$$

The active set used in $F_{t}(x, \alpha)$ at $(x, \alpha) \in R^{n} \times R$ can be written as follows:

$$
Q_{t}(x, \alpha)=\left\{j \in Q \mid f_{j}(x)-\alpha+t>0\right\} .
$$

In [28], a cubic spline smoothing function for $F(x)$ was presented. For any smoothing parameter $t>0$, the active set used in the cubic spline smoothing function at $x \in R^{n}$ can be represented as follows:

$$
Q_{t}(x)=\left\{j \in Q \mid F(x)-f_{j}(x) \leq t\right\} .
$$

In [25], an active set strategy for the aggregate function was introduced. For a given $\epsilon>0$, let

$$
Q_{\epsilon}(x)=\left\{j \in Q \mid F(x)-f_{j}(x) \leq \epsilon\right\} .
$$

Then, the active set $Q^{k}$ used for the aggregate function at $x^{k} \in R^{n}$ is updated as

$$
\begin{aligned}
& Q^{0}=Q_{\epsilon}\left(x^{0}\right), \\
& Q^{k}=Q^{k-1} \cup Q_{\epsilon}\left(x^{k}\right), \quad k \geq 1 .
\end{aligned}
$$

In [26], another active set strategy for the aggregate function was presented. For any smoothing parameter $t>0$, the active set used for the aggregate function at $x \in R^{n}$ is defined as follows:

$$
Q_{t}(x, q)=\left\{j \in Q \mid F(x)-f_{j}(x) \leq \epsilon(t, q)\right\},
$$

where $\epsilon(t, q)>0$ is a complicated combination of several parameters.

In this paper, based on the plus function, an active set smoothing function for the maximum function is proposed, and the smoothing function only relates to a part of component functions, whose function values are close to $F(x)$. It is continuously differentiable, and its gradient is locally Lipschitz continuous and strongly semismooth. Combing the active set smoothing function, a geometric reduction rule for the smoothing parameters, the Armijo line search strategy, the steepest decent direction, and the Newton direction, an active set smoothing method is proposed for solving unconstrained minimax problems. Under the boundedness assumption on the level set of $F(x)$, the convergence of the active set smoothing method is established. Numerical experiments show that the resulting method is stable and efficient, especially for the minimax problems with very many component functions.

The following assumptions and results will be used in this paper:

Assumption 1: the component functions $f_{j}: R^{n} \longrightarrow R$, $j \in Q$, are twice continuously differentiable, and $\nabla f_{j}: R^{n} \longrightarrow R^{n}, j \in Q$, is strongly semismooth

Assumption 2: for any $M>0$, the level set $\Omega_{M}=\left\{x \in R^{n} \mid F(x) \leq M\right\}$ is bounded

Definition 1 (see [29]). Suppose that $\Phi: R^{n} \longrightarrow R^{m}$ is locally Lipschitz continuous, if for any $V \in \partial \Phi(x+h), h \longrightarrow 0$,

$$
V h-\Phi^{\prime}(x ; h)=O\left(\|h\|^{2}\right),
$$

where $\partial \Phi(x)$ is the generalized Jacobian of $\Phi$ at $x, \Phi_{j}^{\prime}(x ; h)$ is the directional derivative of $\Phi_{j}$ at $x$ in the direction $h$ for $j=1, \ldots, m$, and then $\Phi$ is said to be strongly semismooth at $x$.

Lemma 1 (see [29]). Suppose that $\phi: R \longrightarrow R$ and $\psi: R \longrightarrow R$ are strongly semismooth, then

(i) For any $a, b \in R, a \phi+b \psi: R \longrightarrow R$ is strongly semismooth

(ii) $\phi \cdot \psi: R \longrightarrow R$ is strongly semismooth

(iii) If $|\psi(x)| \geq c$ for the constant $c>0, \phi / \psi: R \longrightarrow R$ is strongly semismooth

(iv) $\phi^{\circ} \psi: R \longrightarrow R$ is strongly semismooth

Lemma 2 (see [29]). Suppose that $\Phi: R^{n} \longrightarrow R^{m}$ is locally Lipschitz continuous, if all $\Phi_{i}: R^{n} \longrightarrow R, i=1, \ldots, m$, are strongly semismooth, then $\Phi$ is strongly semismooth.

Lemma 3 (see [30]). For the function $\Phi: R^{n} \longrightarrow R^{m}$, if $\Phi^{\prime}$ is locally Lipschitz continuous, then $\Phi$ is strongly semismooth. 
Theorem 1 (see [24]). Suppose that the component functions $f_{j}: R^{n} \longrightarrow R, j \in Q$, are continuously differentiable. If $x^{*}$ is a local minimizer of problem (1), then

$$
0 \in \partial F\left(x^{*}\right):=\operatorname{conv}_{j \in I\left(x^{*}\right)}\left\{\nabla f_{j}\left(x^{*}\right)\right\},
$$

where

$$
I\left(x^{*}\right):=\left\{j \in Q \mid f_{j}\left(x^{*}\right)=F\left(x^{*}\right)\right\},
$$

and conv $\{A\}$ denotes the convex hull of $A$.

\section{An Active Set Smoothing Function for the Maximum Function}

In this section, based on the plus function $z_{+}: R \longrightarrow R_{+}$:

$$
z_{+}= \begin{cases}z, & z>0 \\ 0, & z \leq 0\end{cases}
$$

we construct the following function $F_{t}^{c}: R^{n} \times R \longrightarrow R$ :

$$
F_{t}^{c}(x, \alpha)=c \alpha+\frac{t}{2} \sum_{j \in Q}\left(1+\frac{f_{j}(x)-c \alpha}{t}\right)_{+}^{2},
$$

where $t>0$ is the smoothing parameter and $c>0$ is the scaling parameter. By the definition of the plus function, Assumption 1, and $t>0$, we have the following result.

Lemma 4. For any $t>0$ and $c>0, F_{t}^{c}: R^{n} \times R \longrightarrow R$ is continuously differentiable.

For any $t>0, c>0$, and $(x, \alpha) \in R^{n} \times R$, let

$$
Q_{t}^{c}(x, \alpha)=\left\{j \in Q \mid f_{j}(x)-c \alpha+t>0\right\},
$$

then we know that

$$
F_{t}^{c}(x, \alpha)=c \alpha+\frac{t}{2} \sum_{j \in Q_{t}^{c}(x, \alpha)}\left(1+\frac{f_{j}(x)-c \alpha}{t}\right)^{2},
$$

only relates to the component functions $f_{j}$ for $j \in Q_{t}^{c}(x, \alpha)$, whose function values are close to $F(x)$. Therefore, $F_{t}^{c}(x, \alpha)$ is called an active set smoothing function for the maximum function in this paper. By direct calculation, we can obtain the gradient of $F_{t}^{c}(x, \alpha)$ :

$$
\nabla F_{t}^{c}(x, \alpha)=\left(\begin{array}{c}
\sum_{j \in Q}\left(1+\frac{f_{j}(x)-c \alpha}{t}\right)_{+} \nabla f_{j}(x) \\
c-c \sum_{j \in Q}\left(1+\frac{f_{j}(x)-c \alpha}{t}\right)_{+}
\end{array}\right),
$$

which can be also written as follows:

$$
\nabla F_{t}^{c}(x, \alpha)=\left(\begin{array}{c}
\sum_{j \in Q_{t}^{c}(x, \alpha)}\left(1+\frac{f_{j}(x)-c \alpha}{t}\right) \nabla f_{j}(x) \\
c-c \sum_{j \in Q_{t}^{c}(x, \alpha)}\left(1+\frac{f_{j}(x)-c \alpha}{t}\right)
\end{array}\right) .
$$

Lemma 5. For any $t>0, c>0$, and $(x, \alpha) \in R^{n} \times R$,

$$
F_{t}^{c}(x, \alpha)>F(x) .
$$

Proof. If $c \alpha>F(x)$, by $t>0$, we have

$$
F_{t}^{c}(x, \alpha)=c \alpha+\frac{t}{2} \sum_{j \in Q}\left(1+\frac{f_{j}(x)-c \alpha}{t}\right)_{+}^{2} \geq c \alpha>F(x) .
$$

If $c \alpha \leq F(x)$, we know $(1+((F(x)-c \alpha) / t))_{+}^{2}=$ $(1+((F(x)-c \alpha) / t))^{2}$ by $t>0$, then we have

$$
\begin{aligned}
F_{t}^{c}(x, \alpha) & =c \alpha+\frac{t}{2} \sum_{j \in Q}\left(1+\frac{f_{j}(x)-c \alpha}{t}\right)_{+}^{2} \\
& \geq c \alpha+\frac{t}{2}\left(1+\frac{F(x)-c \alpha}{t}\right)^{2} \\
& =c \alpha+\frac{t}{2}\left(1+\frac{2(F(x)-c \alpha)}{t}+\frac{(F(x)-c \alpha)^{2}}{t^{2}}\right) \\
& =\frac{t}{2}+F(x)+\frac{(F(x)-c \alpha)^{2}}{2 t} \\
& >F(x) .
\end{aligned}
$$

By (23) and (24), the conclusion holds.

Lemma 6. For any $t>0, c>0, \tau>0$, and $(x, \alpha) \in R^{n} \times R$ satisfying $\left|\nabla_{\alpha} F_{t}^{c}(x, \alpha)\right|<\tau \leq c$,

(i) $F(x)-(\tau / c) t<c \alpha<F(x)+(1-((c-\tau) / c \bar{q})) t$

(ii) $F_{t}^{c}(x, \alpha)<F(x)+\left(1-((c-\tau) / c \bar{q})+\left(\left((c+\tau)^{2}\right) / 2 c^{2}\right)\right) t$, where $\bar{q}=\#\left(Q_{t}^{c}(x, \alpha)\right)$.

Proof. By (10) and $\left|\nabla_{\alpha} F_{t}^{c}(x, \alpha)\right|<\tau$, we know

$$
-\tau<c-c \sum_{j \in Q_{t}^{c}(x, \alpha)}\left(1+\frac{f_{j}(x)-c \alpha}{t}\right)<\tau .
$$

Then, by $c \geq \tau>0$, we have $Q_{t}^{c}(x, \alpha) \neq \varnothing$,

$$
0 \leq 1-\frac{\tau}{c}<\sum_{j \in Q_{t}^{c}(x, \alpha)}\left(1+\frac{f_{j}(x)-c \alpha}{t}\right)<1+\frac{\tau}{c},
$$

and hence, $\max _{j \in Q_{t}^{c}(x, \alpha)}\left(1+\left(\left(f_{j}(x)-c \alpha\right) / t\right)\right)>0$. Therefore, by $t>0$ and $Q_{t}^{c}(x, \alpha) \subseteq Q$, we have

$$
1+\frac{F(x)-c \alpha}{t}=\max _{j \in Q}\left(1+\frac{f_{j}(x)-c \alpha}{t}\right)>0 .
$$

(i) By (26) and (27), we have

$$
\begin{aligned}
\bar{q}\left(1+\frac{F(x)-c \alpha}{t}\right) & \geq \sum_{j \in Q_{t}^{c}(x, \alpha)}\left(1+\frac{f_{j}(x)-c \alpha}{t}\right)>1-\frac{\tau}{c}, \\
1+\frac{F(x)-c \alpha}{t} & \leq \sum_{j \in Q_{t}^{c}(x, \alpha)}\left(1+\frac{f_{j}(x)-c \alpha}{t}\right)<1+\frac{\tau}{c} .
\end{aligned}
$$


Then, by $t>0$, we have

$$
F(x)-\frac{\tau}{c} t<c \alpha<F(x)+\left(1-\frac{c-\tau}{c \bar{q}}\right) t .
$$

(ii) By (26), (29) and $t>0$, we have

$$
\begin{aligned}
F_{t}^{c}(x, \alpha) & =c \alpha+\frac{t}{2} \sum_{j \in Q_{t}^{c}(x, \alpha)}\left(1+\frac{f_{j}(x)-c \alpha}{t}\right)^{2} \\
& \leq c \alpha+\frac{t}{2}\left(\sum_{j \in Q_{t}^{c}(x, \alpha)}\left(1+\frac{f_{j}(x)-c \alpha}{t}\right)\right)^{2} \\
& <F(x)+\left(1-\frac{c-\tau}{c \bar{q}}\right) t+\frac{t}{2}\left(1+\frac{\tau}{c}\right)^{2} \\
& =F(x)+\left(1-\frac{c-\tau}{c \bar{q}}+\frac{(c+\tau)^{2}}{2 c^{2}}\right) t .
\end{aligned}
$$

According to Lemmas 5 and 6 and $(c+\tau)^{2} \leq 4 c^{2}$ for $c \geq \tau>0$, we have the following approximation of $F_{t}^{c}(x, \alpha)$ for $F(x)$.

Lemma 7. For any $t>0, c>0$, and $(x, \alpha) \in R^{n} \times R$ satisfying $\left|\nabla_{\alpha} F_{t}^{c}(x, \alpha)\right|<c$,

(i) $F(x)-t<c \alpha<F(x)+t$

(ii) $F(x)<F_{t}^{c}(x, \alpha)<F(x)+3 t$

For convenience of discussion, for any $t>0, c>0$, and $(x, \alpha) \in R^{n} \times R$, let

$$
z_{+}^{j}(x, \alpha)=\left(1+\frac{f_{j}(x)-c \alpha}{t}\right)_{+}, \quad j \in Q,
$$

then the gradient of $F_{t}^{c}(x, \alpha)$ in (20) can be rewritten as follows:

$$
\nabla F_{t}^{c}(x, \alpha)=\left(\begin{array}{c}
\sum_{j \in Q} z_{+}^{j}(x, \alpha) \nabla f_{j}(x) \\
c-c \sum_{j \in Q} z_{+}^{j}(x, \alpha)
\end{array}\right)
$$

Lemma 8. Suppose that Assumption 1 holds, then for any $t>0$ and $c>0, z_{+}^{j}(x, \alpha): R^{n} \times R \longrightarrow R, j \in Q$, is locally Lipschitz continuous.

Proof. By Assumption 1, we know that $1+\left(\left(f_{j}(x)-\right.\right.$ $(\alpha) / t): R^{n} \times R \longrightarrow R, j \in Q$, is locally Lipschitz continuous with respect to the variables $(x, \alpha)$ for any $t>0$ and $c>0$. By the definition of the plus function, for any $z^{1}, z^{2} \in R$, we know that

(i) If $z^{1} \leq 0, z^{2} \leq 0$, then we have $z_{+}^{1}=z_{+}^{2}=0$, and hence, $\left|z_{+}^{1}-z_{+}^{2}\right|=0$

(ii) If $z^{1} \leq 0, z^{2}>0$, then we have $z_{+}^{1}=0, z_{+}^{2}=z^{2}$, and hence, $\left|z_{+}^{1}-z_{+}^{2}\right|=\left|0-z^{2}\right| \leq\left|z^{1}-z^{2}\right|$ (iii) If $z^{1}>0, z^{2}>0$, then we have $z_{+}^{1}=z^{1}, z_{+}^{2}=z^{2}$, and hence, $\left|z_{+}^{1}-z_{+}^{2}\right|=\left|z^{1}-z^{2}\right|$

Therefore, the plus function is Lipschitz continuous. Hence, $z_{+}^{j}(x, \alpha): R^{n} \times R \longrightarrow R, j \in Q$, is locally Lipschitz continuous.

Lemma 9. Suppose that Assumption 1 holds, then for any $t>0$ and $c>0, \nabla F_{t}^{c}(x, \alpha): R^{n} \times R \longrightarrow R^{n+1}$ is locally Lipschitz continuous.

Proof. By Assumption 1, $\nabla f_{j}(x): R^{n} \longrightarrow R^{n}, j \in Q$, is locally Lipschitz continuous. Then, by Lemma 8, $\sum_{j \in Q} z_{+}^{j}(x, \alpha) \nabla f_{j}(x): R^{n} \times R \longrightarrow R^{n}$ and $c-c \sum_{j \in Q} z_{+}^{j}(x, \alpha)$ : $R^{n} \times R \longrightarrow R$, are locally Lipschitz continuous, which implies that $\nabla F_{t}^{c}(x, \alpha): R^{n} \times R \longrightarrow R^{n+1} \quad$ is locally Lipschitz continuous.

Lemma 10. Suppose that Assumption 1 holds, then for any $t>0$ and $c>0, z_{+}^{j}(x, \alpha): R^{n} \times R \longrightarrow R, j \in Q$, is strongly semismooth.

Proof. By the proof of Lemma 8, the plus function is Lipschitz continuous. For any $h>0$ and $\xi>0$, we know $\xi h>0$ and $h+\xi h>0$, and hence, $h_{+}=h, \quad(\xi h)_{+}=\xi h$, $(h+\xi h)_{+}=h+\xi h ;$ then, we have

$$
\begin{aligned}
\partial(0+h)_{+} & =\{1\}, \\
z_{+}^{\prime}(0 ; h) & =\lim _{\xi \downarrow 0} \frac{(\xi h)_{+}-0}{\xi}=h .
\end{aligned}
$$

For any $h<0$ and $\xi>0$, we know $\xi h<0$ and $h+\xi h<0$, and hence, $h_{+}=(\xi h)_{+}=(h+\xi h)_{+}=0$; then, we have

$$
\begin{aligned}
\partial(0+h)_{+} & =\{0\}, \\
z_{+}^{\prime}(0 ; h) & =\lim _{\xi \downarrow 0} \frac{(\xi h)_{+}-0}{\xi}=0 .
\end{aligned}
$$

Therefore, for any $V \in \partial(0+h)_{+}$and $h \longrightarrow 0$, we have

$$
V h-z_{+}^{\prime}(x ; h)=O\left(\|h\|^{2}\right) \text {, }
$$

which implies that the plus function is strongly semismooth at $z=0$ by Definition 1 . Since the plus function is sufficiently smooth on $R \backslash\{0\}$, we know that the plus function is strongly semismooth on $R$.

By Assumption 1, $\nabla f_{j}(x): R^{n} \longrightarrow R^{n}, j \in Q$, is locally Lipschitz continuous. Then, by Lemma 3, the component functions $f_{j}(x): R^{n} \longrightarrow R, j \in Q$, are strongly semismooth, and hence, $1+\left(\left(f_{j}(x)-c \alpha\right) / t\right): R^{n} \times R \longrightarrow R, j \in Q$, is strongly semismooth with respect to $(x, \alpha)$ for any $t>0$ and $c>0$. Therefore, by (iv) of Lemma $1, z_{+}^{j}(x, \alpha): R^{n} \times R \longrightarrow R$, $j \in Q$, is strongly semismooth.

Lemma 11. Suppose that Assumption 1 holds, then for any $t>0$ and $c>0, \nabla F_{t}^{c}(x, \alpha): R^{n} \times R \longrightarrow R^{n+1}$ is strongly semismooth. 
Proof. By Lemma 9, $\nabla F_{t}^{c}(x, \alpha): R^{n} \times R \longrightarrow R^{n+1}$ is locally Lipschitz continuous. By Assumption $1, \nabla f_{j}(x): R^{n} \longrightarrow R^{n}$, $j \in Q$, is strongly semismooth. By Lemma 10, $z_{+}^{j}(x, \alpha): R^{n} \times R \longrightarrow R, \quad j \in Q$, is strongly semismooth. Then, by (i) and (ii) of Lemma $1, \sum_{j \in Q} z_{+}^{j}(x, \alpha) \nabla f_{j}(x): R^{n} \times$ $R \longrightarrow R^{n}$ and $c-c \sum_{j \in Q} z_{+}^{j}(x, \alpha): R^{n} \times R \longrightarrow R$, are strongly semismooth. Therefore, $\nabla F_{t}^{c}(x, \alpha): R^{n} \times R \longrightarrow R^{n+1}$ is strongly semismooth by Lemma 2 .

For any $(x, \alpha) \in R^{n} \times R$, let $\bar{Q}_{t}^{c}(x, \alpha)=\left\{j \in Q \mid f_{j}(x)-\right.$ $c \alpha+t=0\}, \bar{q}=\#\left(Q_{t}^{c}(x, \alpha)\right)$. By (32) and the definition of the plus function, the Clarke generalized Jacobian of $\nabla F_{t}^{c}(x, \alpha)$ at $(x, \alpha)$ can be represented as follows:

$$
\partial\left(\nabla F_{t}^{c}(x, \alpha)\right)=\left\{\left(\begin{array}{cc}
H_{1}(x, \alpha) & H_{2}(x, \alpha) \\
H_{2}(x, \alpha)^{T} & H_{3}(x, \alpha)
\end{array}\right) \mid \zeta_{j} \in[0,1], j \in \bar{Q}_{t}^{c}(x, \alpha)\right\},
$$

where

$$
\begin{aligned}
& H_{1}(x, \alpha)=\sum_{j \in Q_{t}^{c}(x, \alpha)} z_{+}^{j}(x, \alpha) \nabla^{2} f_{j}(x)+\frac{1}{t}\left(\sum_{j \in \bar{Q}_{t}^{c}(x, \alpha)} \zeta_{j} \nabla f_{j}(x) \nabla f_{j}(x)^{T}+\sum_{j \in Q_{t}^{c}(x, \alpha)} \nabla f_{j}(x) \nabla f_{j}(x)^{T}\right), \\
& H_{2}(x, \alpha)=-\frac{c}{t}\left(\sum_{j \in \bar{Q}_{t}^{c}(x, \alpha)} \zeta_{j} \nabla f_{j}(x)+\sum_{j \in Q_{t}^{c}(x, \alpha)} \nabla f_{j}(x)\right), \\
& H_{3}(x, \alpha)=\frac{c^{2}}{t}\left(\sum_{j \in \bar{Q}_{t}^{c}(x, \alpha)} \zeta_{j}+\bar{q}\right) .
\end{aligned}
$$

For efficient numerical evaluation of $\partial\left(\nabla F_{t}^{c}(x, \alpha)\right)$, we can set $\zeta_{j}=0$ for $j \in \bar{Q}_{t}^{c}(x, \alpha)$; then, we know

$$
V_{t}^{c}(x, \alpha)=\left(\begin{array}{cc}
H_{1}(x, \alpha) & H_{2}(x, \alpha) \\
H_{2}(x, \alpha)^{T} & H_{3}(x, \alpha)
\end{array}\right) \in \partial\left(\nabla F_{t}^{c}(x, \alpha)\right)
$$

where

$$
\begin{aligned}
& H_{1}(x, \alpha)=\sum_{j \in Q_{t}^{c}(x, \alpha)}\left(z_{+}^{j}(x, \alpha) \nabla^{2} f_{j}(x)+\frac{1}{t} \nabla f_{j}(x) \nabla f_{j}(x)^{T}\right), \\
& H_{2}(x, \alpha)=-\frac{c}{t} \sum_{j \in Q_{t}^{c}(x, \alpha)} \nabla f_{j}(x), \\
& H_{3}(x, \alpha)=\frac{c^{2} \bar{q}}{t}
\end{aligned}
$$

\section{An Active Set Smoothing Method and Its Convergence}

In this section, based on the active set smoothing function $F_{t}^{c}(x, \alpha)$ for $F(x)$ and the smoothing methods introduced in [24], an active set smoothing method is proposed to solve problem (1). For a starting point $\left(x^{0}, \alpha^{0}\right) \in R^{n} \times R$ and an initial smoothing parameter $t^{0}>0$, the initial scaling parameter $c^{0}>0$ is chosen from a bounded region in Subroutine 1 , which reduces the ill-conditioning of $V_{t^{0}}^{c^{0}}\left(x^{0}, \alpha^{0} / c^{0}\right)$ caused by the scaling problem of the variable $\alpha \in R$; then, $\alpha^{0,0}$ is set to be $\alpha^{0} / c^{0}$. The Armijo line search strategy, the steepest decent direction, and the Newton direction, in which the selection of the search direction depends on the condition number of $V_{t^{0}}^{c^{0}}\left(x^{k, 0}, \alpha^{k, 0}\right)$ and two convergence conditions for $k \geq 0$, are used to compute an approximate solution $\left(x^{k^{0}, 0}, \alpha^{k^{0}, 0}\right)$ of the smoothing problem $P_{t^{0}}^{c^{0}}$ :

$$
\min _{(x, \alpha) \in R^{n} \times R} F_{t^{0}}^{c^{0}}(x, \alpha) .
$$

Then, the smoothing parameter $t^{0}$ geometrically reduces to $t^{1}$, the scaling parameter $c^{1}$ is chosen from a bounded region in Subroutine $1, \alpha^{k^{0}, 0}$ is updated to $\alpha^{k^{0}+1,1}$ in two ways to balance the efficiency and convergence of the resulting algorithm in Subroutine 1, and the smoothing problem $P_{t^{1}}^{c^{1}}$ :

$$
\min _{(x, \alpha) \in R^{n} \times R} F_{t^{1}}^{c^{1}}(x, \alpha),
$$

is solved with the starting point $\left(x^{k^{0}, 0}, \alpha^{k^{0}+1,1}\right)$. By repeating this process, a sequence of smooth, unconstrained optimization problems is solved. As the smoothing parameters $t^{i}$ go to 0 , a solution of problem (1) can be obtained by the solutions of the smoothing problems $P_{t^{i}}^{c^{i}}$.

Algorithm 1. An active set smoothing algorithm.

Data. Input $\beta, \gamma \in(0,1) ; C_{l} \in(0,1), C_{u}>1, \widehat{t} \in(0,1)$; $\kappa \gg 1, \quad \kappa_{1}^{P}, \kappa_{2}^{P}, \kappa_{1}, \kappa_{2}, \kappa_{3}>0 ; \omega_{t}, \omega_{c} \in(0,1) ; \tau(t):(0, \infty) \longrightarrow$ $(0, \infty)$ satisfying $\lim _{t \rightarrow 0} \tau(t)=0 ; x^{0} \in R^{n}, \alpha^{0} \in R, t^{0}>0$. 
Step 0: set $i=0, k=0, x^{0,0}=x^{0}, \alpha^{0,0}=\alpha^{0}$, and $c^{0}=1$, and go to Subroutine 1 .

Step 1: (compute the search direction) compute the condition number $C^{k, i}$ of $V_{t^{i}}^{c^{i}}\left(x^{k, i}, \alpha^{k, i}\right)$. If $C^{k, i}<\kappa$, compute the Newton direction $d_{t^{i}}^{c^{i}}\left(x^{k, i}, \alpha^{k, i}\right)$ by solving

$$
V_{t^{i}}^{c^{i}}\left(x^{k, i}, \alpha^{k, i}\right) d_{t^{i}}^{c^{i}}\left(x^{k, i}, \alpha^{k, i}\right)+\nabla F_{t^{i}}^{c^{i}}\left(x^{k, i}, \alpha^{k, i}\right)=0 .
$$

If $V_{t^{i}}^{c^{i}}\left(x^{k, i}, \alpha^{k, i}\right)$ is positive and the Newton direction $d_{t^{i}}^{c^{i}}\left(x^{k, i}, \alpha^{k, i}\right)$ satisfies

$$
\begin{gathered}
\left\|d_{t^{i}}^{c^{i}}\left(x^{k, i}, \alpha^{k, i}\right)\right\| \leq \kappa_{1}^{P}\left\|\nabla F_{t^{i}}^{c^{i}}\left(x^{k, i}, \alpha^{k, i}\right)\right\|, \\
\left\langle-\nabla F_{t^{i}}^{c^{i}}\left(x^{k, i}, \alpha^{k, i}\right), d_{t^{i}}^{c^{i}}\left(x^{k, i}, \alpha^{k, i}\right)\right\rangle \geq \kappa_{2}^{P}\left\|\nabla F_{t^{i}}^{c^{i}}\left(x^{k, i}, \alpha^{k, i}\right)\right\|^{2},
\end{gathered}
$$

go to Step 2.

If $V_{t^{i}}^{c^{i}}\left(x^{k, i}, \alpha^{k, i}\right)$ is not positive and the Newton direction $d_{t^{i}}^{c^{i}}\left(x^{k, i}, \alpha^{k, i}\right)$ satisfies

$$
\begin{gathered}
\left\|d_{t^{i}}^{c^{i}}\left(x^{k, i}, \alpha^{k, i}\right)\right\| \leq \kappa_{1}\left\|\nabla F_{t^{i}}^{c^{i}}\left(x^{k, i}, \alpha^{k, i}\right)\right\|, \\
\left\langle-\nabla F_{t^{i}}^{c^{i}}\left(x^{k, i}, \alpha^{k, i}\right), d_{t^{i}}^{c^{i}}\left(x^{k, i}, \alpha^{k, i}\right)\right\rangle \geq \kappa_{2}\left\|\nabla F_{t^{i}}^{c^{i}}\left(x^{k, i}, \alpha^{k, i}\right)\right\|^{2}, \\
\left\langle-\nabla F_{t^{i}}^{c^{i}}\left(x^{k, i}, \alpha^{k, i}\right), d_{t^{i}}^{c^{i}}\left(x^{k, i}, \alpha^{k, i}\right)\right\rangle \geq \kappa_{3}\left\|\nabla F_{t^{i}}^{c^{i}}\left(x^{k, i}, \alpha^{k, i}\right)\right\| \\
\cdot\left\|d_{t^{i}}^{c^{i}}\left(x^{k, i}, \alpha^{k, i}\right)\right\|,
\end{gathered}
$$

go to Step 2 .

Else, compute the steepest decent direction

$$
d_{t^{i}}^{c^{i}}\left(x^{k, i}, \alpha^{k, i}\right)=-\nabla F_{t^{i}}^{c^{i}}\left(x^{k, i}, \alpha^{k, i}\right),
$$

go to Step 2.

Step 2: (compute the stepsize) let $\lambda_{t^{i}}^{c^{i}}\left(x^{k, i}, \alpha^{k, i}\right)=\beta^{l}$, where $l$ is the smallest nonnegative integer satisfying

$$
\begin{aligned}
& F_{t^{i}}^{c^{i}}\left(\left(x^{k, i}, \alpha^{k, i}\right)+\beta^{l} d_{t^{i}}^{c^{i}}\left(x^{k, i}, \alpha^{k, i}\right)\right)-F_{t^{i}}^{c^{i}}\left(x^{k, i}, \alpha^{k, i}\right) \\
& \quad \leq \gamma \beta^{l}\left\langle\nabla F_{t^{i}}^{c^{i}}\left(x^{k, i}, \alpha^{k, i}\right), d_{t^{i}}^{c^{i}}\left(x^{k, i}, \alpha^{k, i}\right)\right\rangle,
\end{aligned}
$$

go to Step 3 .

Step 3: set $\left(x^{k+1, i}, \alpha^{k+1, i}\right)=\left(x^{k, i}, \alpha^{k, i}\right)+\lambda_{t^{i}}^{c^{i}}\left(x^{k, i}, \alpha^{k, i}\right)$ $d_{t^{i}}^{c^{i}}\left(x^{k, i}, \alpha^{k, i}\right)$, replace $k$ by $k+1$, and go to Step 4 .

Step 4: if $\left\|\nabla F_{t^{i}}^{c^{i}}\left(x^{k, i}, \alpha^{k, i}\right)\right\|<\tau\left(t^{i}\right)$, go to Step 5; else, go to Step 1.

Step 5: (adjustment of the smoothing parameter) set $k^{i}=k, x^{k+1, i+1}=x^{k, i}, \quad \alpha^{k+1, i+1}=\alpha^{k, i}, t^{i+1}=\omega_{t} t^{i}$, and $c^{i+1}=c^{i}$, replace $i$ by $i+1$ and $k$ by $k+1$, and go to Subroutine 1.

Subroutine 1: adjustment of the scaling parameter.

Substep 0: set $x=x^{k, i}, t=t^{i}, c^{l}=\omega_{c} c^{i}$, and $c^{u}=c^{i} / \omega_{c}$. If $i=0$ or $t \leq \widehat{t}$, set $\bar{\alpha}^{i}=c^{i} \alpha^{k, i}$ :

$$
\begin{aligned}
& \alpha_{l}^{k, i}=\frac{\bar{\alpha}^{i}}{c^{l}}, \\
& \alpha_{u}^{k, i}=\frac{\bar{\alpha}^{i}}{c^{u}}
\end{aligned}
$$

else, set $\bar{\alpha}^{i}=c^{i} \alpha^{k, i}-\left(1 / \omega_{t}-1\right) t$ :

$$
\begin{aligned}
\alpha_{l}^{k, i} & =\frac{\bar{\alpha}^{i}}{c^{l}}, \\
\alpha^{k, i} & =\frac{\bar{\alpha}^{i}}{c^{i}}, \\
\alpha_{u}^{k, i} & =\frac{\bar{\alpha}^{i}}{c^{u}} .
\end{aligned}
$$

Compute the condition numbers $C^{k, i}$ of $V_{t}^{c^{i}}\left(x, \alpha^{k, i}\right), C_{l}^{k, i}$ of $V_{t}^{c^{l}}\left(x, \alpha_{l}^{k, i}\right)$, and $C_{u}^{k, i}$ of $V_{t}^{c^{u}}\left(x, \alpha_{u}^{k, i}\right)$. If $c^{l} \geq C_{l}$ and $C_{l}^{k, i}<C^{k, i}$, go to Substep 1; else, if $c^{u} \leq C_{u}$ and $C_{u}^{k, i}<C^{k, i}$, go to Substep 2; else, go to Step 4 of Algorithm 1 with $c^{i}$, $C^{k, i}, V_{t}^{c^{i}}\left(x, \alpha^{k, i}\right)$, and $\alpha^{k, i}$.

Substep 1: set $c^{i}=c^{l}, C^{k, i}=C_{l}^{k, i}, V_{t}^{c^{i}}\left(x, \alpha^{k, i}\right)=V_{t}^{c^{l}}(x$, $\left.\alpha_{l}^{k, i}\right)$, and $\alpha^{k, i}=\alpha_{l}^{k, i}$, and go to Substep 3 .

Substep 2: set $c^{i}=c^{u}, C^{k, i}=C_{u}^{k, i}, V_{t}^{c^{i}}\left(x, \alpha^{k, i}\right)=V_{t}^{c^{u}}(x$, $\left.\alpha_{u}^{k, i}\right)$, and $\alpha^{k, i}=\alpha_{u}^{k, i}$, and go to Substep 4 .

Substep 3: set $c^{l}=\omega_{c} c^{l}$ and $\alpha_{l}^{k, i}=\bar{\alpha}^{i} / c^{l}$ and compute the condition number $C_{l}^{k, i}$ of $V_{t}^{c^{l}}\left(x, \alpha_{l}^{k, i}\right)$. If $C_{l}^{k, i}<C^{k, i}$ and $c^{l} \geq C_{l}$, go to Substep 1; else, go to Step 4 of Algorithm 1 with $c^{i}, C^{k, i}, V_{t}^{c^{i}}\left(x, \alpha^{k, i}\right)$, and $\alpha^{k, i}$.

Substep 4: set $c^{u}=c^{u} / \omega_{c}$ and $\alpha_{u}^{k, i}=\bar{\alpha}^{i} / c^{u}$ and compute the condition number $C_{u}^{k, i}$ of $V_{t}^{c^{u}}\left(x, \alpha_{u}^{k, i}\right)$. If $C_{u}^{k, i}<C^{k, i}$ and $c^{u} \leq C_{u}$, go to Substep 2; else, go to Step 4 of Algorithm 1 with $c^{i}, C^{k, i}, V_{t}^{c^{i}}\left(x, \alpha^{k, i}\right)$, and $\alpha^{k, i}$.

Remark 1. In Subroutine 1 for adjusting the scaling parameter $c^{i}$, if $t^{i}>\widehat{t}, \alpha^{k, i}$ is updated to satisfy

$$
c^{i+1} \alpha^{k^{i}+1, i+1}=c^{i} \alpha^{k^{i}, i}-\left(1-\omega_{t}\right) t^{i} .
$$

Then, by $x^{k^{i}+1, i+1}=x^{k^{i}, i}$ and $t^{i+1}=\omega_{t} t^{i}$, we have

$$
\begin{aligned}
& f_{j}\left(x^{k^{i}+1, i+1}\right)-c^{i+1} \alpha^{k^{i}+1, i+1}+t^{i+1}=f_{j}\left(x^{k^{i}, i}\right)-c^{i} \alpha^{k^{i}, i}+t^{i}, \quad j \in Q, \\
& Q_{t^{+1+1}}^{c^{i+1}}\left(x^{k^{i}+1, i+1}, \alpha^{k^{i}+1, i+1}\right)=Q_{t^{i}}^{c^{i}}\left(x^{k^{i, i},}, \alpha^{k^{i, i}}\right), \\
& z_{+}^{j}\left(x^{k^{i}+1, i+1}, \alpha^{k^{i}+1, i+1}\right)=\frac{z_{+}^{j}\left(x^{k^{i}, i}, \alpha^{k^{i}, i}\right)}{\omega_{t}}, \quad j \in Q, \\
& \nabla_{x} F_{t^{i+1}}^{c^{i+1}}\left(x^{k^{i}+1, i+1}, \alpha^{k^{i}+1, i+1}\right)=\frac{\nabla_{x} F_{t^{i}}^{c^{i}}\left(x^{k^{i}, i}, \alpha^{k^{i}, i}\right)}{\omega_{t}}, \\
& \nabla_{\alpha} F_{t^{i+1}}^{i+1}\left(x^{k^{i}+1, i+1}, \alpha^{k^{i}+1, i+1}\right)=c^{i+1}\left(1-\frac{1-\nabla_{\alpha} F_{t^{i}}^{i}\left(x^{k^{i}, i}, \alpha^{k^{i}, i}\right) / c^{i}}{\omega_{t}}\right), \\
& V_{t^{i+1}}^{c^{i+1}}\left(x^{k^{i}+1, i+1}, \alpha^{k^{i}+1, i+1}\right)=\frac{V_{t^{i}}^{c^{i}}\left(x^{k^{i}, i}, \alpha^{k^{, i}, i}\right)}{\omega_{t}} .
\end{aligned}
$$


If $t^{i} \leq \widehat{t}, \alpha^{k, i}$ is updated to satisfy

$$
c^{i+1} \alpha^{k^{i}+1, i+1}=c^{i} \alpha^{k^{i}, i},
$$

which keeps the monotonicity of $\left\{F_{t^{i}}^{c^{i}}\left(x^{k, i}, \alpha^{k, i}\right)\right\}$ with respect to $k$ in Lemma 13.

Theorem 2 (local convergence [31]). For any $t>0$ and $c>0$, suppose that $\left(x^{*}, \alpha^{*}\right)$ is a stationary point of the problem $P_{t}^{c}$. If all $V \in \partial\left(\nabla F_{t}^{c}\left(x^{*}, \alpha^{*}\right)\right)$ are nonsingular, then there exist a neighborhood $N\left(x^{*}, \alpha^{*}\right)$ of $\left(x^{*}, \alpha^{*}\right)$ and a constant $M$ such that for any $(x, \alpha) \in N\left(x^{*}, \alpha^{*}\right)$ and any $V \in \partial\left(\nabla F_{t}^{c}(x, \alpha)\right), V$ is nonsingular and

$$
\left\|V^{-1}\right\| \leq M
$$

The sequence $\left\{\left(x^{k}, \alpha^{k}\right)\right\}$ produced by any initial point $\left(x^{0}, \alpha^{0}\right) \in N\left(x^{*}, \alpha^{*}\right)$ and the semismooth Newton method $\left(x^{k+1}, \alpha^{k+1}\right)=\left(x^{k}, \alpha^{k}\right)-\left(V^{k}\right)^{-1} \nabla F_{t}^{c}\left(x^{k}, \alpha^{k}\right)\left(V^{k} \in \partial\right.$

$\left.\left(\nabla F_{t}^{c}\left(x^{k}, \alpha^{k}\right)\right)\right)$ quadratically converges to $\left(x^{*}, \alpha^{*}\right)$.

Lemma 12. Suppose that Assumption 1 holds, then for any bounded set $S \subset R^{n} \times R$ and parameters $\beta, \gamma \in(0,1), t>0$, and $c>0$, there exists a $\lambda_{S}<\infty$ such that for any $(x, \alpha) \in S$, $\lambda_{t}^{c}(x, \alpha) \geq \lambda_{S}$ and

$$
F_{t}^{c}\left((x, \alpha)+\lambda_{t}^{c}(x, \alpha) d_{t}^{c}(x, \alpha)\right)-F_{t}^{c}(x, \alpha) \leq-\lambda_{s}\left\|\nabla F_{t}^{c}(x, \alpha)\right\|^{2},
$$

where $\lambda_{t}^{c}(x, \alpha)$ is the stepsize computed in Step 2 of Algorithm 1.

Proof. Let $\kappa_{1}^{*}=\max \left\{\kappa_{1}^{P}, \kappa_{1}, 1\right\}$ and $\kappa_{2}^{*}=\min \left\{\kappa_{2}^{P}, \kappa_{2}, 1\right\}$. By (43)-(47), the search direction $d_{t}^{c}(x, \alpha)$ satisfies

$$
\begin{gathered}
\left\|d_{t}^{c}(x, \alpha)\right\| \leq \kappa_{1}^{*}\left\|\nabla F_{t}^{c}(x, \alpha)\right\|, \\
\left\langle-\nabla F_{t}^{c}(x, \alpha), d_{t}^{c}(x, \alpha)\right\rangle \geq \kappa_{2}^{*}\left\|\nabla F_{t}^{c}(x, \alpha)\right\|^{2} .
\end{gathered}
$$

By Lemma 9, $\nabla F_{t}^{c}(x, \alpha): R^{n} \times R \longrightarrow R^{n+1}$ is locally Lipschitz continuous, and then, there exists a Lipschitz constant $L_{S}>0$ such that for any $\left(x^{1}, \alpha^{1}\right),\left(x^{2}, \alpha^{2}\right) \in S$,

$$
\left\|\nabla F_{t}^{c}\left(x^{1}, \alpha^{1}\right)-\nabla F_{t}^{c}\left(x^{2}, \alpha^{2}\right)\right\| \leq L_{S}\left\|\left(x^{1}, \alpha^{1}\right)-\left(x^{2}, \alpha^{2}\right)\right\| .
$$

For any $(x, \alpha) \in S$ and $\lambda \in(0,1]$, by the mean value theorem, there exists a $\xi \in(0,1)$ such that

$$
\begin{aligned}
F_{t}^{c} & \left((x, \alpha)+\lambda d_{t}^{c}(x, \alpha)\right)-F_{t}^{c}(x, \alpha)-\gamma \lambda\left\langle\nabla F_{t}^{c}(x, \alpha), d_{t}^{c}(x, \alpha)\right\rangle \\
& =\left\langle\nabla F_{t}^{c}\left((x, \alpha)+\xi \lambda d_{t}^{c}(x, \alpha)\right), \lambda d_{t}^{c}(x, \alpha)\right\rangle-\gamma \lambda\left\langle\nabla F_{t}^{c}(x, \alpha), d_{t}^{c}(x, \alpha)\right\rangle \\
& =\lambda(1-\gamma)\left\langle\nabla F_{t}^{c}(x, \alpha), d_{t}^{c}(x, \alpha)\right\rangle+\left\langle\nabla F_{t}^{c}\left((x, \alpha)+\xi \lambda d_{t}^{c}(x, \alpha)\right)-\nabla F_{t}^{c}(x, \alpha), \lambda d_{t}^{c}(x, \alpha)\right\rangle \\
& \leq \lambda(1-\gamma)\left\langle\nabla F_{t}^{c}(x, \alpha), d_{t}^{c}(x, \alpha)\right\rangle+\lambda\left\|\nabla F_{t}^{c}\left((x, \alpha)+\xi \lambda d_{t}^{c}(x, \alpha)\right)-\nabla F_{t}^{c}(x, \alpha)\right\|\left\|d_{t}^{c}(x, \alpha)\right\| \\
& \leq \lambda(1-\gamma)\left\langle\nabla F_{t}^{c}(x, \alpha), d_{t}^{c}(x, \alpha)\right\rangle+\lambda^{2} \xi L_{S}\left\|d_{t}^{c}(x, \alpha)\right\|^{2} \\
& \leq-\lambda\left(\kappa_{2}^{*}(1-\gamma)-\kappa_{1}^{* 2} \lambda L_{S}\right)\left\langle\nabla F_{t}^{c}(x, \alpha)\right\rangle^{2},
\end{aligned}
$$

where the second inequality comes from (58) and the last inequality comes from (56), (57), and $\xi \in(0,1)$. Let

$$
\lambda^{*}=\min \left\{1, \frac{\kappa_{2}^{*}(1-\gamma)}{\kappa_{1}^{* 2} L_{S}}\right\},
$$

then it follows from (59) that for any $\lambda \in\left(0, \lambda^{*}\right]$,

$$
F_{t}^{c}\left((x, \alpha)+\lambda d_{t}^{c}(x, \alpha)\right)-F_{t}^{c}(x, \alpha)-\gamma \lambda\left\langle\nabla F_{t}^{c}(x, \alpha), d_{t}^{c}(x, \alpha)\right\rangle \leq 0,
$$

and hence, $\lambda_{t}^{c}(x, \alpha) \geq \beta \lambda^{*}$. Therefore, by (57), we have

$$
\begin{aligned}
& F_{t}^{c}\left((x, \alpha)+\lambda_{t}^{c}(x, \alpha) d_{t}^{c}(x, \alpha)\right)-F_{t}^{c}(x, \alpha) \\
& \quad \leq \gamma \lambda_{t}^{c}(x, \alpha)\left\langle\nabla F_{t}^{c}(x, \alpha), d_{t}^{c}(x, \alpha)\right\rangle \\
& \quad \leq-\beta \gamma \lambda^{*} \kappa_{2}^{*}\left\|\nabla F_{t}^{c}(x, \alpha)\right\|^{2} .
\end{aligned}
$$

Then, by $\gamma, \kappa_{2}^{*} \in(0,1]$, the conclusion holds for

$$
\lambda_{S}=\min \left\{\beta \gamma \kappa_{2}^{*}, \frac{\beta \gamma(1-\gamma)\left(\kappa_{2}^{*}\right)^{2}}{\left(\kappa_{1}^{*}\right)^{2} L_{S}}\right\} .
$$

Lemma 13. Suppose that Assumptions 1 and 2 hold and $\tau\left(t^{i}\right) \leq \omega_{t} c^{i}$, then for any $\left(x^{0}, \alpha^{0}\right) \in R^{n} \times R, t^{0}>0$ and $c^{0}>0$, the sequence $\left\{\left(x^{k, i}, \alpha^{k, i}\right)\right\}$ generated by Algorithm 1 satisfies the following:

(i) For any $i \geq 0,\left\{F_{t^{i}}^{c^{i}}\left(x^{k, i}, \alpha^{k, i}\right)\right\}$ is monotone decreasing with respect to $k$

(ii) There exists an $\hat{i}$ such that the sequence $\left\{F_{t^{i}}^{c^{i}}\left(x^{k, i}\right.\right.$, $\left.\left.\alpha^{k, i}\right)\right\}_{i>i}$ is monotone decreasing with respect to $k$

(iii) The sequence $\left\{x^{k, i}\right\}$ is bounded

(iv) The sequence $\left\{\alpha^{k, i}\right\}$ is bounded

Proof.

(i) For any $i \geq 0$ and $\left(x^{k, i}, \alpha^{k, i}\right) \in R^{n} \times R$ satisfying $\left\|d_{t^{i}}^{c^{i}}\left(x^{k, i}, \alpha^{k, i}\right)\right\| \geq \tau\left(t^{i}\right)$, by Lemma 12 , there exists a $l^{k, i} \geq 0$ such that

$$
\begin{aligned}
& F_{t^{i}}^{c^{i}}\left(x^{k+1, i}, \alpha^{k+1, i}\right)-F_{t^{i}}^{c^{i}}\left(x^{k, i}, \alpha^{k, i}\right) \\
& \quad \leq \gamma \beta^{\beta^{k, i}}\left\langle\nabla F_{t^{i}}^{c^{i}}\left(x^{k, i}, \alpha^{k, i}\right), d_{t^{i}}^{c^{i}}\left(x^{k, i}, \alpha^{k, i}\right)\right\rangle<0,
\end{aligned}
$$


then we know

$$
F_{t^{i}}^{c^{i}}\left(x^{k+1, i}, \alpha^{k+1, i}\right)<F_{t^{i}}^{c^{i}}\left(x^{k, i}, \alpha^{k, i}\right),
$$

which implies that the conclusion holds.

(ii) By $t^{i+1}=\omega_{t} t^{i}$ and $\omega_{t} \in(0,1)$, for any $t^{0}>0$ and $\widehat{t} \in(0,1)$, there exists an $\widehat{i}$ such that for any $i \geq \widehat{i}$,

$$
t^{i} \leq \hat{t}
$$

For any $i \geq \hat{i}$, if $\left\|\nabla F_{t^{i}}^{c^{i}}\left(x^{k, i}, \alpha^{k, i}\right)\right\|<\tau\left(t^{i}\right)$, by the definition of $\nabla F_{t}^{c}(x, \alpha)$ in (20), we have

$-\tau\left(t^{i}\right)<c^{i}-c^{i} \sum_{j \in Q}\left(1+\frac{f_{j}\left(x^{k, i}\right)-c^{i} \alpha^{k, i}}{t^{i}}\right)_{+}<\tau\left(t^{i}\right)$.

Then, by $\tau\left(t^{i}\right) \leq \omega_{t} c^{i}<c^{i}$ and $t^{i}>0$, we have

$$
1+\frac{F\left(x^{k, i}\right)-c^{i} \alpha^{k, i}}{t^{i}}=\max _{j \in Q}\left(1+\frac{f_{j}\left(x^{k, i}\right)-c^{i} \alpha^{k, i}}{t^{i}}\right)_{+}>0 .
$$

Let $t$ be the variable of the function $F_{t}^{c}(x, \alpha)$ for given $(x, \alpha) \in R^{n} \times R$ and $c>0$, which is redefined as $F_{x, \alpha}^{c}(t)$, then by the mean value theorem, there exists a $\bar{t} \in\left(\omega_{t} t^{i}, t^{i}\right)$ such that

$F_{x^{k, i}, \alpha^{k, i}}^{c^{i}}\left(t^{i}\right)-F_{x^{k, i}, \alpha^{k, i}}^{c^{i}}\left(\omega_{t} t^{i}\right)=\left(F_{x^{k, i}, \alpha^{k, i}}^{c^{i}}\right)^{\prime}(\bar{t})\left(t^{i}-\omega_{t} t^{i}\right)$,

with

$\left(F_{x, \alpha}^{c}\right)^{\prime}(t)=\frac{1}{2} \sum_{j \in Q}\left(1+\frac{f_{j}(x)-c \alpha}{t}\right)_{+}\left(1-\frac{f_{j}(x)-c \alpha}{t}\right)$.

If $F\left(x^{k, i}\right) \leq c^{i} \alpha^{k, i}$, by $\bar{t}>0$, we have

$$
1-\frac{f_{j}\left(x^{k, i}\right)-c^{i} \alpha^{k, i}}{\bar{t}}>0, \quad j \in Q
$$

and hence $\left(F_{x^{k, i}, \alpha^{k, i}}^{c^{i}}\right)^{\prime}(\bar{t})>0$ by (68). If $F\left(x^{k, i}\right)>c^{i} \alpha^{k, i}$, by (67) and (68) and $\tau\left(t^{i}\right) \leq \omega_{t} c^{i}$, we have

$$
\begin{aligned}
c^{i} & -c^{i}\left(1+\frac{F\left(x^{k, i}\right)-c^{i} \alpha^{k, i}}{t^{i}}\right) \\
& \geq c^{i}-c^{i} \sum_{j \in Q}\left(1+\frac{f_{j}\left(x^{k, i}\right)-c^{i} \alpha^{k, i}}{t^{i}}\right)_{+} \\
> & -\tau\left(t^{i}\right) \geq-\omega_{t} c^{i},
\end{aligned}
$$

which implies

$$
\frac{F\left(x^{k, i}\right)-c^{i} \alpha^{k, i}}{t^{i}}<\omega_{t} .
$$

By $\bar{t}>\omega_{t} t^{i}>0$, we have

$$
\begin{aligned}
& \frac{F\left(x^{k, i}\right)-c^{i} \alpha^{k, i}}{\bar{t}}<\frac{F\left(x^{k, i}\right)-c^{i} \alpha^{k, i}}{\omega_{t} t^{i}}<1, \\
& \frac{f_{j}\left(x^{k, i}\right)-c^{i} \alpha^{k, i}}{\bar{t}} \leq \frac{F\left(x^{k, i}\right)-c^{i} \alpha^{k, i}}{\bar{t}}<1, \quad j \in Q .
\end{aligned}
$$

Then, we know that (71) also holds and hence $\left(F_{x^{k, i}, \alpha^{k, i}}^{c^{i}}\right)^{\prime}(\bar{t})>0$ by (68). Therefore, for the case of $\left\|\nabla F_{t^{i}}^{c^{i}}\left(x^{k, i}, \alpha^{k, i}\right)\right\|<\tau\left(t^{i}\right)$, by (69), we have

$$
F_{x^{k, i}, \alpha^{k, i}}^{c^{i}}\left(t^{i}\right)-F_{x^{k, i}, \alpha^{k, i}}^{c^{i}}\left(\omega_{t} t^{i}\right)>0,
$$

which means

$$
F_{t^{i}}^{c^{i}}\left(x^{k, i}, \alpha^{k, i}\right)-F_{\omega_{t} t^{i}}^{c^{i}}\left(x^{k, i}, \alpha^{k, i}\right)>0
$$

By $x^{k+1, i+1}=x^{k, i}$ and $c^{i+1} \alpha^{k+1, i+1}=c^{i} \alpha^{k, i}$ according to (53) and (66), we have

$$
F_{t^{i+1}}^{c^{i+1}}\left(x^{k+1, i+1}, \alpha^{k+1, i+1}\right)=F_{t^{i+1}}^{c^{i}}\left(x^{k, i}, \alpha^{k, i}\right) .
$$

Then, by (76) and $t^{i+1}=\omega_{t} t^{i}$, we know

$$
F_{t^{i+1}}^{c^{i+1}}\left(x^{k+1, i+1}, \alpha^{k+1, i+1}\right)<F_{t^{i}}^{c^{i}}\left(x^{k, i}, \alpha^{k, i}\right) .
$$

Therefore, the sequence $\left\{F_{t^{i}}^{c^{i}}\left(x^{k, i}, \alpha^{k, i}\right)\right\}_{i \geq \hat{i}}$ is monotone decreasing with respect to $k$ by $(65)$ and (78).

(iii) By (i), for any $0 \leq k \leq k^{0}$, we have

$$
F_{t^{0}}^{c^{0}}\left(x^{k, 0}, \alpha^{k, 0}\right) \leq F_{t^{0}}^{c^{0}}\left(x^{0,0}, \alpha^{0,0}\right)
$$

and for any $1 \leq i<\widehat{i}, k^{i-1}+1 \leq k \leq k^{i}$, we have

$$
F_{t^{i}}^{c^{i}}\left(x^{k, i}, \alpha^{k, i}\right) \leq F_{t^{i}}^{c^{i}}\left(x^{k^{i-1}+1, i}, \alpha^{k^{i-1}+1, i}\right) .
$$

By (ii), for any $i \geq \hat{i}$ and $k^{i-1}+1 \leq k \leq k^{i}$,

$$
F_{t^{i}}^{c^{i}}\left(x^{k, i}, \alpha^{k, i}\right) \leq F_{t^{i}}^{c^{\hat{i}}}\left(x^{k^{i-1}+1, \hat{i}}, \alpha^{k^{i-1}+1, \hat{i}}\right) \text {. }
$$

By the finiteness of $i$ satisfying $i<\widehat{i}$, (79), (80), and (81), there exists a constant $F^{0} \in R$ such that for any $\left(x^{k, i}, \alpha^{k, i}\right)$,

$\left(x^{k, i}, \alpha^{k, i}\right) \in \Omega^{F^{0}}=\left\{(x, \alpha) \in R^{n} \times R \mid F_{t}^{c}(x, \alpha) \leq F^{0}\right\}$.

Suppose that for any $M>0$, there exists a point $\left(x^{\bar{k}, \bar{i}}, \alpha^{\bar{k}, \bar{i}}\right) \in \Omega^{F^{0}} \quad$ satisfying $\quad F\left(x^{\bar{k}, \bar{i}}\right)>M$. If $c^{\bar{i}} \alpha^{\bar{k}, \bar{i}} \geq M-t^{\bar{i}}$, by the definition of the plus function and $0<t^{\bar{i}} \leq t^{0}$, we have

$$
F_{t^{i}}^{c^{\bar{i}}}\left(x^{\bar{k}, \bar{i}}, \alpha^{\bar{k}, \bar{i}}\right) \geq c^{\bar{i}} \alpha^{\bar{k}, \bar{i}} \geq M-t^{\bar{i}} \geq M-t^{0} .
$$

If $c^{\bar{i}} \alpha^{\bar{k}, \bar{i}}<M-t^{\bar{i}}$, by $F\left(x^{\bar{k}, \bar{i}}\right)>M$ and $t^{\bar{i}}>0$, we have

$$
1+\frac{F\left(x^{\bar{k}, \bar{i}}\right)-c^{\bar{i}} \alpha^{\bar{k}, \bar{i}}}{t^{\bar{i}}}>1+\frac{M-c^{\bar{i}} \alpha^{\bar{k}, \bar{i}}}{t^{\bar{i}}}>2,
$$


and hence $\left(1+\left(\left(F\left(x^{\bar{k}, \bar{i}}\right)-c^{\bar{i}} \alpha^{\bar{k}, \bar{i}}\right) / t^{\bar{i}}\right)\right)_{+}=1+$ $\left(\left(F\left(x^{\bar{k}, \bar{i}}\right)-c^{\bar{i}} \alpha^{\bar{k}, \bar{i}}\right) / t^{\bar{i}}\right)$ and $\left(1+\left(\left(M-c^{\bar{i}} \alpha^{\bar{k}, \bar{i}}\right) / t^{\bar{i}}\right)\right)_{+}=$ $1+\left(\left(M-c^{\bar{i}} \alpha^{\bar{k}, \bar{i}}\right) / t^{\bar{i}}\right)$. Then, we know

$$
\begin{aligned}
F_{t^{i}}^{c^{i}}\left(x^{\bar{k}, \bar{i}}, \alpha^{\bar{k}, \bar{i}}\right) & =c^{\bar{i}} \alpha^{\bar{k}, \bar{i}}+\frac{t^{\bar{i}}}{2} \sum_{j \in Q}\left(1+\frac{f_{j}\left(x^{\bar{k}, \bar{i}}\right)-c^{\bar{i}} \alpha^{\bar{k}, \bar{i}}}{t^{\bar{i}}}\right)_{+}^{2} \\
& \geq c^{\bar{i}} \alpha^{\bar{k}, \bar{i}}+\frac{t^{\bar{i}}}{2}\left(1+\frac{F\left(x^{\bar{k}, \bar{i}}\right)-c^{\bar{i}} \alpha^{\bar{k}, \bar{i}}}{t^{\bar{i}}}\right)^{2} \\
& >c^{\bar{i}} \alpha^{\bar{k}, \bar{i}}+\frac{t^{\bar{i}}}{2}\left(1+\frac{M-c^{\bar{i}} \alpha^{\bar{k}, \bar{i}}}{t^{\bar{i}}}\right)^{2} \\
& >c^{\bar{i}} \alpha^{\bar{k}, \bar{i}}+t^{\bar{i}}\left(1+\frac{M-c^{\bar{i}} \alpha^{\bar{k}, \bar{i}}}{t^{\bar{i}}}\right) \\
& >M .
\end{aligned}
$$

However, the arbitrariness of $M,(83)$, and (85) contradict $x^{\bar{k}, \bar{i}} \in \Omega^{F^{0}}$; then, there must exist a $M^{*}>0$ such that $F\left(x^{k, i}\right) \leq M^{*}$ for any $F\left(x^{k, i}\right) \leq M^{*}$ and $i \geq 0$. Therefore, we have that $\left\{x^{k, i}\right\} \subseteq \Omega_{M^{*}}$, and hence, the sequence $\left\{x^{k, i}\right\}$ is bounded by Assumption 2 .

(iv) For any $\left(x^{k, i}, \alpha^{k, i}\right)$ with $k \geq 0$ and $i \geq 0$, if $Q_{t^{i}}^{c^{i}}\left(x^{k, i}, \alpha^{k, i}\right)=\varnothing$, by the definition of $Q_{t}^{c}(x, \alpha)$, we know $\left(1+\left(\left(f_{j}\left(x^{k, i}\right)-c^{i} \alpha^{k, i}\right) / t^{i}\right)\right)_{+}=0$ for any $j \in Q$ by $t^{i}>0$. Then, by $\left(x^{k, i}, \alpha^{k, i}\right) \in \Omega^{F^{0}}$, we have

$$
F_{t^{i}}^{c^{i}}\left(x^{k, i}, \alpha^{k, i}\right)=c^{i} \alpha^{k, i} \leq F^{0} .
$$

Hence, by $c^{i} \geq C_{l}>0$, we know

$$
\alpha^{k, i} \leq \frac{F^{0}}{c^{i}} \leq \frac{F^{0}}{C_{l}} .
$$

If $Q_{t^{i}}^{c^{i}}\left(x^{k, i}, \alpha^{k, i}\right) \neq \varnothing$, by $F\left(x^{k, i}\right) \leq M^{*}$ from (iii), we have

$$
t^{i}+M^{*}-c^{i} \alpha^{k, i} \geq t^{i}+F\left(x^{k, i}\right)-c^{i} \alpha^{k, i}>0 .
$$

Then, by $0<t^{i} \leq t^{0}$ and $c^{i} \geq C_{l}>0$, we know

$$
\alpha^{k, i}<\frac{t^{i}+M^{*}}{c^{i}} \leq \frac{t^{0}+M^{*}}{c^{i}} \leq \frac{t^{0}+M^{*}}{C_{l}} .
$$
(89).

Therefore, the sequence $\left\{\alpha^{k, i}\right\}$ is bounded by (87) and

Lemma 14. Suppose that Assumptions 1 and 2 hold, then for any $\left(x^{0}, \alpha^{0}\right) \in R^{n} \times R$ and $t^{0}>0$, the sequences $\left\{\left(x^{k, i}, \alpha^{k, i}\right)\right\}$ and $\left\{t^{i}\right\}$ generated by Algorithm 1 satisfy the following:

(i) For any $i \geq 0$, there exists a $k^{i} \in N$ such that $\left\|\nabla F_{t^{i}}^{c^{i}}\left(x^{k^{i}, i}, \alpha^{k^{i}, i}\right)\right\|<\tau\left(t^{i}\right)$

(ii) The sequence $\left\{t^{i}\right\}$ is infinite and strictly monotone decreasing, $t^{i} \longrightarrow 0$ as $k \longrightarrow \infty$
Proof.

(i) If there exists an $\bar{i} \geq 0$ such that the sequence $\left\{\left(x^{k, \bar{i}}, \alpha^{k, \bar{i}}\right)\right\}$ is infinite, then we have $\| \nabla F_{t^{i}}^{c^{i}}\left(x^{k, \bar{i}}\right.$, $\left.\alpha^{k, \bar{i}}\right) \| \geq \tau\left(t^{\bar{i}}\right)$ and $k \longrightarrow \infty$. By Lemma 12 and the boundedness of $\left\{\left(x^{k, \bar{i}}, \alpha^{k, \bar{i}}\right)\right\}$ from Lemma 13, there exists a constant $C^{i}>0$ such that

$$
\begin{aligned}
& F_{t^{i}}^{c^{i}}\left(x^{k+1, \bar{i}}, \alpha^{k+1, \bar{i}}\right)-F_{t^{i}}^{c^{i}}\left(x^{k, \bar{i}}, \alpha^{k, \bar{i}}\right) \\
& \quad \leq-C^{\bar{i}}\left\|\nabla F_{t^{i}}^{c^{i}}\left(x^{k, \bar{i}}, \alpha^{k, \bar{i}}\right)\right\|^{2} \leq-C^{\bar{i}} \tau\left(t^{\bar{i}}\right)^{2}<0 .
\end{aligned}
$$

Then, by $k \longrightarrow \infty$, we have

$$
\lim _{k \longrightarrow \infty} F_{\bar{t}^{i}}^{c^{i}}\left(x^{k, \bar{i}}, \alpha^{k, \bar{i}}\right)=-\infty,
$$

which contradicts that $\left\{\left(x^{k, \bar{i}}, \alpha^{k, \bar{i}}\right)\right\}$ is bounded and $F_{t^{i}}^{c^{i}}(x, \alpha)$ is continuous on $R^{n} \times R$. Therefore, for any $i \stackrel{t^{i}}{\geq}$, there exists a $k^{i} \in N$ such that $\| \nabla F_{t^{i}}^{c^{i}}\left(x^{k^{i}, i}\right.$, $\left.\alpha^{k^{i}, i}\right) \|<\tau\left(t^{i}\right)$.

(ii) By (i), for any $i \geq 0$, there exists a $k^{i} \in N$ such that $\left\|\nabla F_{t^{i}}^{c^{i}}\left(x^{k^{i}, i}, \alpha^{k^{i}, i}\right)\right\|<\tau\left(t^{i}\right)$, then $t^{i}$ is updated to $t^{i+1}=$ $\omega_{t} t^{i}$. Therefore, by $\omega_{t} \in(0,1)$, we know that $i \longrightarrow \infty$ as $k \longrightarrow \infty$, and hence, the sequence $\left\{t^{i}\right\}$ is infinite and strictly monotone decreasing, $t^{i} \longrightarrow 0$ as $k \longrightarrow \infty$.

Theorem 3. Suppose that Assumptions 1 and 2 hold, $\left\{\left(x^{k, i}, \alpha^{k, i}\right)\right\}$ is the sequence generated by Algorithm 1 , then for any accumulation point $\left(x^{*}, \alpha^{*}\right) \in R^{n} \times R$ of $\left\{\left(x^{k^{i}, i}, \alpha^{k^{i}, i}\right)\right\}$, $0 \in \partial F\left(x^{*}\right)$, i.e., $x^{*}$ is a stationary point of problem (1).

Proof. By Lemmas 13 and 14, the sequence $\left\{\left(x^{k^{i}, i}\right.\right.$, $\left.\left.\alpha^{k^{i}, i}\right)\right\} \subseteq\left\{\left(x^{k, i}, \alpha^{k, i}\right)\right\}$ is infinite and bounded; then, there exists at least one accumulation point of $\left\{\left(x^{k^{i}, i}, \alpha^{k^{,}, i}\right)\right\}$. For any accumulation point $\left(x^{*}, \alpha^{*}\right)$ of $\left\{\left(x^{k^{i}, i}, \alpha^{k^{i}, i}\right)\right\}$, there exists a subsequence of $\left\{\left(x^{k^{i}, i}, \alpha^{k^{i}, i}\right)\right\}$ (denoted also by $\left\{\left(x^{k^{i}, i}, \alpha^{k^{i}, i}\right)\right\}$ for convenience) converging to $\left(x^{*}, \alpha^{*}\right)$. By Lemma 14 , $\left\|\nabla F_{t^{i}}^{c^{i}}\left(x^{k^{i}, i}, \alpha^{k^{i}, i}\right)\right\|<\tau\left(t^{i}\right)$ and $\lim _{t \longrightarrow 0} \tau(t)=0$, we have

$$
\lim _{i \longrightarrow \infty}\left\|\nabla F_{t^{i}}^{c^{i}}\left(x^{k^{i}, i}, \alpha^{k^{i}, i}\right)\right\|=0 .
$$

It follows from (32) and (92) that

$$
\begin{aligned}
\lim _{i \longrightarrow \infty} \sum_{j \in Q} z_{+}^{j}\left(x^{k^{i}, i}, \alpha^{k^{i}, i}\right) \nabla f_{j}\left(x^{k^{i}, i}\right) & =0, \\
\lim _{i \longrightarrow \infty} \sum_{j \in Q} z_{+}^{j}\left(x^{k^{i}, i}, \alpha^{k^{i}, i}\right) & =1 .
\end{aligned}
$$

By Lemma $7,\left(x^{k^{i}, i}, \alpha^{k^{i}, i}\right) \longrightarrow\left(x^{*}, \alpha^{*}\right), i \longrightarrow \infty$, and $t^{i} \longrightarrow 0$, we have

$$
\lim _{i \longrightarrow \infty} F\left(x^{k^{i}, i}\right)=\lim _{i \longrightarrow \infty} c^{i} \alpha^{k^{i}, i}=F\left(x^{*}\right) .
$$

Then, by Assumption 1, we have 


$$
\lim _{i \longrightarrow \infty}\left(f_{j}\left(x^{k^{i}, i}\right)-c^{i} \alpha^{k^{i}, i}\right)=f_{j}\left(x^{*}\right)-F\left(x^{*}\right)<0, \quad j \notin I\left(x^{*}\right) .
$$

Hence, by the finiteness of indexes in $Q \backslash I\left(x^{*}\right)$, there exists an $i_{0}>0$ such that for any $i>i_{0}$,

$$
f_{j}\left(x^{k^{i}, i}\right)-c^{i} \alpha^{k^{i}, i}<-t^{i}, \quad j \notin I\left(x^{*}\right),
$$

which implies

$$
\lim _{i \longrightarrow \infty} z_{+}^{j}\left(x^{k^{i}, i}, \alpha^{k^{i}, i}\right)=0, \quad j \notin I\left(x^{*}\right) .
$$

By (94), $z_{+}^{j}\left(x^{k^{i}, i}, \alpha^{k^{i}, i}\right) \geq 0$ for $j \in I\left(x^{*}\right)$, and by the finiteness of indexes in $I\left(x^{*}\right)$, there exists a subsequence of $\left\{\left(x^{k^{i}, i}, \alpha^{k^{i}, i}\right)\right\}$ (denoted also by $\left\{\left(x^{k^{i}, i}, \alpha^{k^{i}, i}\right)\right\}$ for convenience) and $z_{j}^{*} \in[0,1]$ for $j \in I\left(x^{*}\right)$, such that

$$
\begin{aligned}
& \sum_{j \in I\left(x^{*}\right)} z_{j}^{*}=1, \\
& \lim _{i \longrightarrow \infty} z_{+}^{j}\left(x^{k^{i}, i}, \alpha^{k^{i}, i}\right)=z_{j}^{*} \in[0,1], \\
& j \in I\left(x^{*}\right) .
\end{aligned}
$$

Therefore, by $x^{k^{i}, i} \longrightarrow x^{*}$, (93), (98), (99), and Assumption 1 , we have

$$
\begin{aligned}
& \sum_{j \in I\left(x^{*}\right)} z_{j}^{*} \nabla f_{j}\left(x^{*}\right)=0, \\
& \sum_{j \in I\left(x^{*}\right)} z_{j}^{*}=1, z_{j}^{*} \geq 0, j \in I\left(x^{*}\right),
\end{aligned}
$$

which implies that $x^{*}$ is a stationary point of problem (1).

\section{Numerical Experiments}

In this section, we present the numerical results of Algorithm 1 and several related algorithms for solving unconstrained minimax problems. Algorithm 1 is recorded as ASSF. Fminimax is the MATLAB algorithm "fminimax". Fmincon is the MATLAB algorithm "fmincon" applied to

$$
\min _{(x, \alpha) \in R^{n+1}}\left\{\alpha \mid f_{j}(x)-\alpha \leq 0, j \in Q\right\},
$$

which is equivalent to problem (1). To show the efficiency of the proposed active set smoothing function, we replace it by some other smoothing techniques in Algorithm 1 to obtain several smoothing methods. AF, SSF, and SPF are constructed by Algorithm 1 with aggregate function (2), cubic spline smoothing function introduced in [28], and exact penalty function technique introduced in [18], respectively. TAF and ASAF are constructed by Algorithm 1 and the aggregate function with the active set strategies introduced in $[25,26]$, respectively.

The parameters in Algorithm 1 are set as follows:

$$
\begin{aligned}
\beta & =0.8, \gamma=0.5 ; C_{l}=10^{-2}, C_{u}=10^{2}, \widehat{t}=10^{-3} ; \\
\kappa & =10^{10}, \kappa_{1}^{P}=\kappa_{1}=10^{5}, \kappa_{2}^{P}=\kappa_{2}=10^{-10}, \kappa_{3}=0.25 \\
\omega_{t} & =0.1, \omega_{c}=0.1, \tau\left(t^{i}\right)=10^{-3} .
\end{aligned}
$$

For the moderately sized test problems, $t^{0}$ and $\alpha^{0}$ are set as follows:

$$
\begin{aligned}
& t^{0}=\sum_{j \in Q} f_{j}\left(x^{0}\right)-q \min _{j \in Q} f_{j}\left(x^{0}\right)+1, \\
& \alpha^{0}=\frac{\sum_{j \in Q} f_{j}\left(x^{0}\right)}{c^{0} q}+\frac{t^{0}}{c^{0}}-\frac{t^{0}}{c^{0} q},
\end{aligned}
$$

and then, we have

$$
\begin{aligned}
Q_{t^{0}}^{c^{0}}\left(x^{0}, \alpha^{0}\right) & =Q, \\
\sum_{j \in Q}\left(1+\frac{f_{j}\left(x^{0}\right)-c^{0} \alpha^{0}}{t^{0}}\right)_{+} & =1,
\end{aligned}
$$

which implies that $\nabla_{x} F_{t^{0}}^{c^{0}}\left(x^{0}, \alpha^{0}\right)$ is a convex combination of the gradients of all the component functions. For the test problems with very many component functions, $t^{0}$ is set as

$$
t^{0}=\max \left\{1, \frac{q\left(F\left(x^{0}\right)-\min _{j \in Q} f_{j}\left(x^{0}\right)\right)}{10}\right\} .
$$

$\alpha^{0} \in\left(F\left(x^{0}\right)-t^{0}, F\left(x^{0}\right)+t^{0}\right)$ is computed by the bisection method according to

$$
\left|1-\sum_{j \in Q}\left(1+\frac{f_{j}\left(x^{0}\right)-c^{0} \alpha^{0}}{t^{0}}\right)_{+}\right|<0.1 \text {. }
$$

For the algorithm ASAF, the parameter $\epsilon$ in (10) is set as

$$
\epsilon=0.8\left(F(x)-\min _{j \in Q} f_{j}(x)\right),
$$

for the moderately sized test problems, and

$$
\epsilon=0.4\left(F(x)-\min _{j \in Q} f_{j}(x)\right),
$$

for the test examples with very many component functions. For the algorithm TAF, the parameter $\epsilon(t, q)$ in (12) is set as

$$
\epsilon(t, q)=t \ln \left(\max \left\{1, \frac{\left(2 \epsilon_{1}-\epsilon_{2}\right)(q-1)}{\epsilon_{2}},\left(\frac{2 \epsilon_{3}+6 \epsilon_{1}^{2}}{t-\epsilon_{4}}\right) \frac{(q-1)}{\epsilon_{4}}\right\}\right),
$$

with $\epsilon_{1}=0.1, \epsilon_{2}=0.01, \epsilon_{3}=0.01$, and $\epsilon_{4}=0.1$. For the algorithms AF, ASAF, TAF, SSF, and SPF, the initial smoothing parameters are set to $t^{0}=1$. The termination criterion for the algorithm AF is set as

$$
\begin{aligned}
t & <10^{-3}, \\
\left\|\nabla F_{t}(x)\right\| & \leq 10^{-3} .
\end{aligned}
$$


Table 1: The CPU time for Examples 1-10.

\begin{tabular}{|c|c|c|c|c|c|c|c|c|c|}
\hline Ex. & $q$ & ASSF & SSF & SPF & TAF & ASAF & $\mathrm{AF}$ & Fminimax & Fmincon \\
\hline \multirow{3}{*}{1} & $10^{5}$ & 0.1035 & 0.7598 & 1.2153 & 0.3283 & 0.2872 & 0.2924 & 34.7621 & 226.4573 \\
\hline & $10^{6}$ & 0.8800 & 7.5375 & 7.3612 & 3.2775 & 2.8821 & 3.0391 & 2204.3730 & fail $^{3}$ \\
\hline & $10^{7}$ & 10.7318 & 75.3454 & 75.8656 & 32.6120 & 28.2999 & 31.4882 & fail $^{3}$ & $\mathrm{fail}^{3}$ \\
\hline \multirow{3}{*}{2} & $10^{5}$ & 0.0862 & 0.0965 & 0.1222 & 0.0954 & 0.2447 & 0.0909 & 7.1100 & 116.8411 \\
\hline & $10^{6}$ & 0.5647 & 0.9765 & 2.8985 & 0.9576 & 3.1395 & 0.9704 & 116.5674 & 1491.4805 \\
\hline & $10^{7}$ & 9.1149 & 10.7622 & 26.5794 & 9.7821 & 32.9736 & 9.4956 & 1487.9868 & fail $^{3}$ \\
\hline \multirow{3}{*}{3} & $10^{5}$ & 1.4902 & 4.4586 & 27.7835 & 4.4605 & 4.0761 & 7.7392 & 122.9883 & fail $^{2}$ \\
\hline & $10^{6}$ & 19.8937 & 59.5392 & 839.3762 & 68.2482 & 55.0242 & 103.4272 & 3044.0625 & $\mathrm{fail}^{2}$ \\
\hline & $10^{7}$ & 203.0458 & 514.4458 & 3569.5139 & 676.4006 & 501.4630 & 926.5762 & $\mathrm{fail}^{3}$ & $\mathrm{fail}^{2}$ \\
\hline \multirow{3}{*}{4} & $10^{5}$ & 0.3202 & 0.5836 & 4.5430 & 0.6396 & 2.1124 & 2.1355 & 69.3814 & fail $^{2}$ \\
\hline & $10^{6}$ & 3.4366 & 7.9853 & 44.6778 & 8.8451 & 27.3115 & 27.6770 & 1545.7716 & $\mathrm{fail}^{2}$ \\
\hline & $10^{7}$ & 36.0206 & 71.4938 & 645.1772 & 78.9203 & 230.0224 & 237.1365 & fail $^{3}$ & $\mathrm{fail}^{2}$ \\
\hline \multirow{3}{*}{5} & $10^{5}$ & 0.9396 & 2.6478 & 9.6803 & 3.7163 & 3.2187 & 3.7483 & 230.2623 & fail $^{1}$ \\
\hline & $10^{6}$ & 16.4395 & 31.1775 & 92.1263 & 45.2874 & 43.4843 & 45.5968 & $\mathrm{fail}^{3}$ & fail $^{1}$ \\
\hline & $10^{7}$ & 158.8988 & 307.4791 & 1002.5981 & 432.1560 & 401.5995 & 436.5213 & $\mathrm{fail}^{3}$ & fail $^{1}$ \\
\hline \multirow{3}{*}{6} & $10^{5}$ & 0.6966 & 3.7246 & 3.1238 & 4.4810 & 3.4686 & 4.5208 & 42.9713 & fail $^{3}$ \\
\hline & $10^{6}$ & 7.2618 & 49.6754 & 46.5345 & 59.6989 & 46.1585 & 60.0291 & 2912.3607 & fail $^{3}$ \\
\hline & $10^{7}$ & 74.8332 & 478.8652 & 455.7022 & 561.2579 & 425.4030 & 566.2980 & $\mathrm{fail}^{3}$ & $\mathrm{fail}^{3}$ \\
\hline \multirow{3}{*}{7} & $10^{5}$ & 1.1056 & 7.0048 & 13.4588 & 3.2790 & 9.3249 & 3.2450 & 169.5213 & fail $^{2}$ \\
\hline & $10^{6}$ & 26.3445 & 74.6292 & 177.1446 & 35.2551 & 99.2012 & 34.5205 & $\mathrm{fail}^{3}$ & fail $^{2}$ \\
\hline & $10^{7}$ & 123.4221 & 749.7656 & 1834.9277 & 343.0618 & 984.0483 & 341.2569 & fail $^{3}$ & fail $^{2}$ \\
\hline \multirow{3}{*}{8} & $10^{5}$ & 0.9361 & 1.8597 & 8.1073 & 1.3515 & 2.6522 & 1.5489 & 157.1865 & fail $^{2}$ \\
\hline & $10^{6}$ & 12.6151 & 25.1838 & 86.3288 & 19.5087 & 35.3458 & 20.1976 & fail $^{3}$ & fail $^{2}$ \\
\hline & $10^{7}$ & 108.6392 & 236.4630 & 949.2546 & 184.0629 & 328.1475 & 189.5273 & fail $^{3}$ & fail $^{2}$ \\
\hline \multirow{3}{*}{9} & $10^{5}$ & 0.4863 & 1.5641 & 10.0722 & 1.4539 & 0.7162 & 1.4945 & 21.4071 & fail $^{3}$ \\
\hline & $10^{6}$ & 8.0179 & 18.8813 & 174.8562 & 18.4460 & 9.4945 & 18.7385 & 283.0764 & fail $^{3}$ \\
\hline & $10^{7}$ & 58.8060 & 177.5352 & 2275.1924 & 162.9317 & 101.1354 & 165.5642 & 3543.2556 & fail $^{3}$ \\
\hline \multirow{3}{*}{10} & $10^{3}$ & 0.9273 & 1.9002 & 1.1572 & 14.5336 & 28.9550 & 30.5158 & 1.3285 & fail $^{2}$ \\
\hline & $10^{4}$ & 0.6413 & 19.4758 & 10.9090 & 97.0372 & 177.8060 & 186.0173 & 14.5788 & fail $^{2}$ \\
\hline & $10^{5}$ & 1.6718 & 183.3566 & 129.7202 & 471.6195 & 792.5688 & 809.8969 & 131.9020 & fail $^{2}$ \\
\hline
\end{tabular}

The termination criteria for the algorithms ASSF, SSF, SPF, ASAF, and TAF are set as

$$
\begin{aligned}
t & <10^{-3}, \\
\left\|\nabla \mathscr{F}_{t}(x)\right\| & \leq 10^{-3},
\end{aligned}
$$

or

$$
F(x) \leq F\left(x_{A F}^{*}\right)
$$

where $\nabla \mathscr{F}_{t}(x)$ represents the gradient of the smoothing function with respect to the variable $x$ and $x_{A F}^{*}$ is the approximation solution computed by the algorithm AF. The numerical results were obtained by running MATLAB R2014a on a laptop with Inter(R) Core(TM) i5-7300HQ CPU 2.50GHZ and 4.00 GB memory.

We carry out a comparison on three categories of test problems described in the Appendix. The first category of problems, Examples 1-10, emanates from the discretized semi-infinite minimax problems, and the number of the component functions is at least 1000 . The second category of problems, Example 11, possesses many variables and many component functions. The third category of problems, Examples $12-45$, is composed by various moderately sized test problems. Tables 1-3 list the CPU time; Tables 4-6 list the number of function evaluations and iterations; Tables 7 and 8 list the average proportion of the component functions used in the active set strategy; the word fail $^{1}$ means that the stepsize cannot be computed in the region $\left[10^{-10}, 1\right]$; the word $\mathrm{fail}^{2}$ means that the number of iterations in Fminimax or Fmincon reaches the upper limit; the word $\mathrm{fail}^{3}$ means that the CPU time exceeds 3600 seconds. In order to make the advantages of Algorithm 1 clearer and more explicit, the corresponding Dolan-Morée performance profiles proposed in [32] are shown in Figures 1-3 for three categories of examples above.

For all the test problems with very many component functions, we see that Algorithm 1 is predominantly faster than other algorithms from Tables 1 and 2 and Figures 1 and 2 , the proposed active set strategy results in more significant reduction of gradient evaluations than the active set strategies in $[18,25,26,28]$ from Tables 7 and 8, and Fminimax and Fmincon have poor stability and low efficiency. For most moderately sized test problems, we see from Tables 3 and 6 and Figure 3 that Algorithm 1 requires fewer iterations and function evaluations and takes less CPU time than the other algorithms considered. 
TABle 2: The CPU time for Example 11.

\begin{tabular}{lccccccccc}
\hline$n$ & $q$ & ASSF & SSF & SPF & TAF & ASAF & AF & Fminimax & Fmincon \\
\hline $10^{2}$ & $10^{2}$ & $\mathbf{0 . 5 2 7 5}$ & 0.7747 & 12.2677 & 0.7556 & 0.7715 & 0.7928 & 464.3152 & $\mathrm{fail}^{3}$ \\
$10^{2}$ & $10^{3}$ & $\mathbf{2 . 7 3 6 9}$ & 15.8849 & 76.2165 & 14.4669 & 15.1963 & 15.2076 & $\mathrm{fail}^{3}$ & $\mathrm{fail}^{3}$ \\
$10^{2}$ & $10^{4}$ & $\mathbf{2 5 . 7 5 5 0}$ & 587.5630 & fail $^{3}$ & 533.8570 & 615.3645 & 665.1596 & $\mathrm{fail}^{3}$ & $\mathrm{fail}^{3}$ \\
10 & $10^{3}$ & $\mathbf{0 . 2 1 2 7}$ & 2.4103 & 2.8646 & 2.3061 & 2.3695 & 2.4319 & 2.8159 & 20.2106 \\
$10^{2}$ & $10^{3}$ & $\mathbf{2 . 7 1 4 1}$ & 12.0482 & 74.3301 & 10.7229 & 10.0013 & 11.2506 & $\mathrm{fail}^{3}$ & $\mathrm{fail}^{3}$ \\
$10^{3}$ & $10^{3}$ & $\mathbf{8 0 6 . 9 2 0 6}$ & 2286.4695 & fail $^{3}$ & 2186.7536 & 2098.2569 & 2304.5143 & $\mathrm{fail}^{3}$ & $\mathrm{fail}^{3}$ \\
\hline
\end{tabular}

TABle 3: The CPU time for Examples 12-45.

\begin{tabular}{|c|c|c|c|c|c|c|c|c|}
\hline Ex. & ASSF & SSF & SPF & TAF & ASAF & $\mathrm{AF}$ & Fminimax & Fmincon \\
\hline 12 & 0.0018 & 0.0026 & 0.1070 & 0.0013 & 0.0023 & 0.0017 & 0.0636 & 0.0569 \\
\hline 13 & 0.0011 & 0.0012 & 0.0167 & 0.0021 & 0.0037 & 0.0045 & 0.0931 & 0.0578 \\
\hline 14 & 0.2468 & 0.4617 & 0.6172 & 0.2469 & 0.4213 & 0.0013 & 0.0959 & $\mathrm{fail}^{2}$ \\
\hline 15 & 0.0190 & 0.0132 & 0.1243 & 0.0087 & 0.0117 & 0.0125 & 1.4777 & fail $^{2}$ \\
\hline 16 & 0.0437 & 0.0316 & 2.3675 & 0.0298 & 0.0353 & 0.0297 & 5.8776 & $\mathrm{fail}^{2}$ \\
\hline 17 & 0.0099 & 0.0193 & 0.3882 & 0.0129 & 0.0137 & 0.0193 & 0.8454 & 0.1955 \\
\hline 18 & 0.0376 & 0.0417 & 0.8257 & 0.0839 & 0.0849 & 0.0702 & 1.8279 & fail $^{2}$ \\
\hline 19 & 0.1895 & 0.7442 & 0.5214 & 0.8614 & 0.9749 & 0.8750 & 0.5122 & 0.6373 \\
\hline 20 & 1.8828 & 3.6656 & 3.0255 & 3.5755 & 3.8257 & 3.5837 & 3.0208 & 3.0462 \\
\hline 21 & 0.0054 & 0.4821 & fail $^{1}$ & 0.0178 & 0.0229 & 0.0198 & 0.0845 & 0.1491 \\
\hline 22 & 0.0074 & 0.1384 & 3.0235 & 0.0169 & 0.0312 & 0.0228 & 0.0321 & 0.0607 \\
\hline 23 & 0.0113 & 0.8615 & 0.3981 & 0.0432 & 0.0588 & 0.0481 & 0.0897 & 0.2281 \\
\hline 24 & 0.0026 & 0.0881 & 0.0248 & 0.0073 & 0.0092 & 0.0077 & 0.0211 & 0.0360 \\
\hline 25 & 1.7941 & 16.1289 & 6.0794 & 6.2755 & 11.2465 & 12.2501 & 6.1201 & 6.2704 \\
\hline 26 & 0.0006 & 0.0057 & 0.0096 & 0.0190 & 0.0088 & 0.0051 & 0.1165 & 0.0926 \\
\hline 27 & 0.0990 & 0.1668 & 1.7366 & 0.8320 & 1.1020 & 0.7201 & 0.4449 & 0.0894 \\
\hline 28 & 0.0018 & 0.0055 & 0.0147 & 0.0038 & 0.0044 & 0.0032 & 0.0126 & 0.0179 \\
\hline 29 & 0.0010 & 0.0022 & 0.0126 & 0.0011 & 0.0027 & 0.0007 & 0.0180 & 0.0247 \\
\hline 30 & 0.0015 & 0.0036 & 0.0093 & 0.0016 & 0.0040 & 0.0025 & 0.0101 & 0.0170 \\
\hline 31 & 0.0005 & 0.0010 & 0.0014 & 0.0009 & 0.0007 & 0.0006 & 0.0177 & 0.0185 \\
\hline 32 & 0.0018 & 0.0055 & 0.1108 & 0.0041 & 0.0055 & 0.0038 & 0.0101 & 0.0193 \\
\hline 33 & 0.0017 & 0.0089 & 0.0165 & 0.0038 & 0.0060 & 0.0040 & 0.0101 & 0.0174 \\
\hline 34 & 0.0004 & 0.0017 & 0.0031 & 0.0016 & 0.0019 & 0.0015 & 0.0106 & 0.0184 \\
\hline 35 & 0.0018 & 0.0061 & 0.0206 & 0.0051 & 0.0063 & 0.0047 & 0.0087 & 0.0184 \\
\hline 36 & 0.0008 & 0.0026 & 0.0093 & 0.0018 & 0.0028 & 0.0018 & 0.0082 & 0.0148 \\
\hline 37 & 0.0022 & 0.0060 & 0.0210 & 0.0076 & 0.0097 & 0.0073 & 0.0160 & 0.0310 \\
\hline 38 & 0.0030 & 0.0053 & 0.5716 & 0.0027 & 0.0037 & 0.0018 & 0.0543 & 0.1928 \\
\hline 39 & 0.0038 & 0.0090 & 0.0263 & 0.0106 & 0.0089 & 0.0064 & 0.0327 & 0.0910 \\
\hline 40 & 0.0228 & 0.0291 & 0.1652 & 0.0611 & 0.1229 & 0.1114 & 0.0113 & 0.9211 \\
\hline 41 & 0.0001 & 0.0053 & 0.0061 & 0.0047 & 0.0064 & 0.0051 & 0.0145 & 0.0203 \\
\hline 42 & 0.0018 & 0.0093 & 0.0127 & 0.0064 & 0.0176 & 0.0094 & 0.0068 & 0.8627 \\
\hline 43 & 0.0022 & 0.0046 & 0.0590 & 0.0050 & 0.0063 & 0.0047 & 0.0157 & 0.0216 \\
\hline 44 & 0.0045 & 0.0107 & 0.0505 & 0.0117 & 0.0157 & 0.0122 & 0.0168 & 0.0572 \\
\hline 45 & 0.0331 & fail $^{1}$ & 2.6303 & 0.5594 & 0.1648 & 21.6874 & 0.0681 & fail $^{1}$ \\
\hline
\end{tabular}

TABLe 4: The number of function evaluations and iterations for Examples 1-10.

\begin{tabular}{|c|c|c|c|c|c|c|c|}
\hline Ex. & $q$ & ASSF & SSF & SPF & TAF & ASAF & $\mathrm{AF}$ \\
\hline \multirow{3}{*}{1} & $10^{5}$ & $(34,23)$ & $(67,14)$ & $(188,77)$ & $(93,15)$ & $(79,14)$ & $(84,15)$ \\
\hline & $10^{6}$ & $(27,20)$ & $(246,22)$ & $(88,34)$ & $(85,15)$ & $(79,14)$ & $(84,15)$ \\
\hline & $10^{7}$ & $(33,23)$ & $(343,21)$ & $(97,40)$ & $(83,14)$ & $(79,14)$ & $(84,15)$ \\
\hline \multirow{3}{*}{2} & $10^{5}$ & $(23,18)$ & $(30,13)$ & $(19,19)$ & $(32,10)$ & $(55,13)$ & $(32,10)$ \\
\hline & $10^{6}$ & $(23,18)$ & $(34,15)$ & $(60,35)$ & $(32,10)$ & $(55,13)$ & $(32,10)$ \\
\hline & $10^{7}$ & $(32,22)$ & $(34,13)$ & $(16,16)$ & $(32,10)$ & $(55,13)$ & $(32,10)$ \\
\hline \multirow{3}{*}{3} & $10^{5}$ & $(242,101)$ & $(564,118)$ & $(1381,928)$ & $(737,219)$ & $(580,109)$ & $(812,262)$ \\
\hline & $10^{6}$ & $(238,103)$ & $(761,137)$ & $(4852,4518)$ & $(798,245)$ & $(583,108)$ & $(1039,266)$ \\
\hline & $10^{7}$ & $(239,103)$ & $(765,141)$ & $(4852,4518)$ & $(804,249)$ & $(583,108)$ & $(819,262)$ \\
\hline
\end{tabular}


TABle 4: Continued.

\begin{tabular}{|c|c|c|c|c|c|c|c|}
\hline Ex. & $q$ & ASSF & SSF & SPF & TAF & ASAF & $\mathrm{AF}$ \\
\hline \multirow{3}{*}{4} & $10^{5}$ & $(60,50)$ & $(242,53)$ & $(808,385)$ & $(503,33)$ & $(466,35)$ & $(610,47)$ \\
\hline & $10^{6}$ & $(66,58)$ & $(270,59)$ & $(1008,258)$ & $(657,41)$ & $(550,38)$ & $(614,48)$ \\
\hline & $10^{7}$ & $(71,63)$ & $(296,68)$ & $(1408,271)$ & $(632,46)$ & $(634,40)$ & $(599,47)$ \\
\hline \multirow{3}{*}{5} & $10^{5}$ & $(84,58)$ & $(76,45)$ & $(407,233)$ & $(281,65)$ & $(277,63)$ & $(284,67)$ \\
\hline & $10^{6}$ & $(93,61)$ & $(87,50)$ & $(407,233)$ & $(280,65)$ & $(276,63)$ & $(284,68)$ \\
\hline & $10^{7}$ & $(89,59)$ & $(98,55)$ & $(407,233)$ & $(280,65)$ & $(276,63)$ & $(284,68)$ \\
\hline \multirow{3}{*}{6} & $10^{5}$ & $(53,39)$ & $(293,216)$ & $(274,95)$ & $(296,229)$ & $(231,231)$ & $(299,231)$ \\
\hline & $10^{6}$ & $(53,39)$ & $(293,216)$ & $(396,124)$ & $(296,229)$ & $(231,231)$ & $(299,231)$ \\
\hline & $10^{7}$ & $(53,39)$ & $(293,216)$ & $(426,123)$ & $(296,229)$ & $(231,231)$ & $(299,231)$ \\
\hline \multirow{3}{*}{7} & $10^{5}$ & $(19,16)$ & $(130,28)$ & $(379,111)$ & $(55,17)$ & $(342,68)$ & $(55,17)$ \\
\hline & $10^{6}$ & $(67,39)$ & $(128,33)$ & $(498,138)$ & $(55,17)$ & $(362,72)$ & $(55,17)$ \\
\hline & $10^{7}$ & $(44,19)$ & $(125,31)$ & $(507,137)$ & $(55,17)$ & $(364,74)$ & $(55,17)$ \\
\hline \multirow{3}{*}{8} & $10^{5}$ & $(66,39)$ & $(145,57)$ & $(606,180)$ & $(214,39)$ & $(550,52)$ & $(215,40)$ \\
\hline & $10^{6}$ & $(60,38)$ & $(131,61)$ & $(567,180)$ & $(216,40)$ & $(572,54)$ & $(216,40)$ \\
\hline & $10^{7}$ & $(54,33)$ & $(137,56)$ & $(801,227)$ & $(215,40)$ & $(593,56)$ & $(215,40)$ \\
\hline \multirow{3}{*}{9} & $10^{5}$ & $(79,14)$ & $(47,21)$ & $(434,152)$ & $(48,18)$ & $(39,15)$ & $(48,18)$ \\
\hline & $10^{6}$ & $(79,17)$ & $(59,21)$ & $(677,200)$ & $(48,18)$ & $(39,15)$ & $(48,18)$ \\
\hline & $10^{7}$ & $(76,11)$ & $(49,20)$ & $(935,270)$ & $(48,18)$ & $(39,15)$ & $(48,18)$ \\
\hline \multirow{3}{*}{10} & $10^{3}$ & $(745, \mathbf{6 3})$ & $(1071,103)$ & $(243,107)$ & $(107139,4270)$ & $(111873,4454)$ & $(135709,5096)$ \\
\hline & $10^{4}$ & $(74,53)$ & $(1094,105)$ & $(278,120)$ & $(134725,5136)$ & $(137748,5240)$ & $(136931,5213)$ \\
\hline & $10^{5}$ & $(69,49)$ & $(2477,495)$ & $(423,140)$ & $(122067,4649)$ & $(112489,4968)$ & $(122803,4677)$ \\
\hline
\end{tabular}

TABLE 5: The number of function evaluations and iterations for Example 11.

\begin{tabular}{lccccccc}
\hline$n$ & $q$ & ASSF & SSF & SPF & TAF & ASAF \\
\hline $10^{2}$ & $10^{2}$ & $(212,41)$ & $(196,38)$ & $(1110,919)$ & $(180, \mathbf{3 2})$ & $(174,31)$ & $(\mathbf{1 7 5}, \mathbf{3 2})$ \\
$10^{2}$ & $10^{3}$ & $(\mathbf{2 4 5}, \mathbf{5 5})$ & $(561,65)$ & $(1010,378)$ & $(553,62)$ & $(557,64)$ & $(557,64)$ \\
$10^{2}$ & $10^{4}$ & $(\mathbf{3 1 8} \mathbf{8 5})$ & $(1952,143)$ & fail & $(1707,114)$ & $(1888,139)$ & $(2857,166)$ \\
10 & $10^{3}$ & $(\mathbf{2 0 8}, \mathbf{4 5})$ & $(392,49)$ & $(5658,249)$ & $(391,49)$ & $(392,49)$ & $(395,48)$ \\
$10^{2}$ & $10^{3}$ & $(\mathbf{2 2 8}, \mathbf{4 7})$ & $(464,59)$ & $(983,381)$ & $(459,56)$ & $(561,62)$ & $(459,56)$ \\
$10^{3}$ & $10^{3}$ & $(\mathbf{2 5 5}, \mathbf{5 9})$ & $(562,63)$ & fail $^{3}$ & $(563,65)$ & $(559,60)$ & $(559,60)$ \\
\hline
\end{tabular}

TABLE 6: The number of function evaluations and iterations for Examples 12-45.

\begin{tabular}{|c|c|c|c|c|c|c|}
\hline Ex. & ASSF & SSF & SPF & TAF & ASAF & $\mathrm{AF}$ \\
\hline 12 & $(26,19)$ & $(29,13)$ & $(56,41)$ & $(24,11)$ & $(27,12)$ & $(36,16)$ \\
\hline 13 & $(9,5)$ & $(5,5)$ & $(93,48)$ & $(7,5)$ & $(6,5)$ & $(7,5)$ \\
\hline 14 & $(5054,2352)$ & $(5024,2342)$ & $(6058,6052)$ & $(5195,2341)$ & $(5027,2335)$ & $(33,9)$ \\
\hline 15 & $(12,6)$ & $(5,5)$ & $(135,76)$ & $(7,5)$ & $(6,5)$ & $(7,5)$ \\
\hline 16 & $(14,6)$ & $(6,6)$ & $(494,264)$ & $(7,5)$ & $(7,6)$ & $(7,5)$ \\
\hline 17 & $(13,6)$ & $(4,4)$ & $(316,143)$ & $(10,5)$ & $(10,5)$ & $(11,6)$ \\
\hline 18 & $(14,6)$ & $(6,6)$ & $(180,92)$ & $(22,9)$ & $(22,7)$ & $(16,6)$ \\
\hline 19 & $(5466,213)$ & $(11827,582)$ & $(664,586)$ & $(23561,649)$ & $(23700,649)$ & $(24435,671)$ \\
\hline 20 & $(41561,2428)$ & $(46979,2844)$ & $(3103,3056)$ & $(88377,2981)$ & $(87642,2846)$ & $(89606,3016)$ \\
\hline 21 & $(69,36)$ & $(3296,3186)$ & fail $^{1}$ & $(618,40)$ & $(655,42)$ & $(649,47)$ \\
\hline 22 & $(121,40)$ & $(2508,353)$ & $(22442,21961)$ & $(695,55)$ & $(844,61)$ & $(740,58)$ \\
\hline 23 & $(68,41)$ & $(12151,694)$ & $(4623,1197)$ & $(1277,55)$ & $(1339,81)$ & $(1181,82)$ \\
\hline 24 & $(21,19)$ & $(638,279)$ & $(203,116)$ & $(121,27)$ & $(111,26)$ & $(119,30)$ \\
\hline 25 & $(26994,1525)$ & $(101048,5186)$ & $(5215,5126)$ & $(135661,5598)$ & $(220821,7685)$ & $(261684,8977)$ \\
\hline 26 & $(9,5)$ & $(22,11)$ & $(65,44)$ & $(151,119)$ & $(145,13)$ & $(145,13)$ \\
\hline 27 & $(237,237)$ & $(356,239)$ & $(3691,2763)$ & $(16060,878)$ & $(15714,852)$ & $(10800,622)$ \\
\hline 28 & $(30,18)$ & $(148,21)$ & $(164,135)$ & $(104,19)$ & $(92,19)$ & $(95,19)$ \\
\hline 29 & $(13,11)$ & $(26,10)$ & $(105,104)$ & $(11,8)$ & $(12,10)$ & $(8,8)$ \\
\hline 30 & $(31,15)$ & $(104,14)$ & $(84,71)$ & $(45,10)$ & $(71,16)$ & $(71,16)$ \\
\hline 31 & $(8,8)$ & $(8,6)$ & $(7,9)$ & $(7,6)$ & $(6,4)$ & $(10,6)$ \\
\hline
\end{tabular}


TABle 6: Continued.

\begin{tabular}{|c|c|c|c|c|c|c|}
\hline Ex. & ASSF & SSF & SPF & TAF & ASAF & $\mathrm{AF}$ \\
\hline 32 & $(28,18)$ & $(178,18)$ & $(1145,1053)$ & $(119,22)$ & $(115,20)$ & $(119,22)$ \\
\hline 33 & $(27,18)$ & $(270,22)$ & $(256,117)$ & $(112,19)$ & $(118,25)$ & $(118,25)$ \\
\hline 34 & $(4,4)$ & $(19,10)$ & $(33,22)$ & $(30,11)$ & $(44,7)$ & $(39,13)$ \\
\hline 35 & $(30,17)$ & $(209,19)$ & $(259,175)$ & $(171,20)$ & $(150,21)$ & $(153,24)$ \\
\hline 36 & $(12,8)$ & $(42,10)$ & $(39,21)$ & $(56,7)$ & $(56,10)$ & $(53,10)$ \\
\hline 37 & $(25,21)$ & $(158,18)$ & $(225,163)$ & $(228,26)$ & $(214,25)$ & $(218,29)$ \\
\hline 38 & $(21,13)$ & $(64,12)$ & $(2090,2073)$ & $(38,8)$ & $(33,10)$ & $(20,7)$ \\
\hline 39 & $(\mathbf{3 1}, 22)$ & $(142,22)$ & $(174,114)$ & $(262,17)$ & $(103,22)$ & $(105,24)$ \\
\hline 40 & $(966,45)$ & $(592,90)$ & $(4612,232)$ & $(2341,192)$ & $(4278,214)$ & $(4549,213)$ \\
\hline 41 & $(18,18)$ & $(165,39)$ & $(53,38)$ & $(97,37)$ & $(107,26)$ & $(119,38)$ \\
\hline 42 & $(26,15)$ & $(236,36)$ & $(180,78)$ & $(158,44)$ & $(469,52)$ & $(306,41)$ \\
\hline 43 & $(23,21)$ & $(165,46)$ & $(601,502)$ & $(175,49)$ & $(175,28)$ & $(167,42)$ \\
\hline 44 & $(36,28)$ & $(228,52)$ & $(360,228)$ & $(307,53)$ & $(339,32)$ & $(304,46)$ \\
\hline 45 & $(195,26)$ & fail $^{1}$ & $(6172,3109)$ & $(1145,35)$ & $(4754,174)$ & $(225933,4256)$ \\
\hline
\end{tabular}

TABLE 7: The proportion of the component functions used in the active set strategy for Examples 1-10.

\begin{tabular}{|c|c|c|c|c|c|c|}
\hline Ex. & $q$ & ASSF & SSF & SPF & TAF & ASAF \\
\hline \multirow{3}{*}{1} & $10^{5}$ & 0.4992 & 0.7292 & 0.6553 & 0.7582 & 0.9666 \\
\hline & $10^{6}$ & 0.4241 & 0.7280 & 0.6497 & 0.7666 & 0.9666 \\
\hline & $10^{7}$ & 0.4987 & 0.7001 & 0.6178 & 0.8056 & 0.9666 \\
\hline \multirow{3}{*}{2} & $10^{5}$ & 0.4264 & 0.7737 & 0.8901 & 0.7370 & 0.8956 \\
\hline & $10^{6}$ & 0.4264 & 0.7528 & 0.8811 & 0.7497 & 0.8956 \\
\hline & $10^{7}$ & 0.5306 & 0.7793 & 0.8820 & 0.7616 & 0.8956 \\
\hline \multirow{3}{*}{3} & $10^{5}$ & 0.8052 & 0.9965 & 0.7298 & 0.9848 & 0.9948 \\
\hline & $10^{6}$ & 0.8069 & 0.9204 & 0.5178 & 0.9870 & 0.9947 \\
\hline & $10^{7}$ & 0.8069 & 0.9129 & 0.5178 & 0.9880 & 0.9946 \\
\hline \multirow{3}{*}{4} & $10^{5}$ & 0.1952 & 0.3062 & 0.7900 & 0.6262 & 0.9549 \\
\hline & $10^{6}$ & 0.1672 & 0.3020 & 0.7049 & 0.6334 & 0.9584 \\
\hline & $10^{7}$ & 0.1540 & 0.3009 & 0.7037 & 0.6301 & 0.9543 \\
\hline \multirow{3}{*}{5} & $10^{5}$ & 0.4633 & 0.8963 & 0.9309 & 0.8392 & 0.9843 \\
\hline & $10^{6}$ & 0.4878 & 0.8325 & 0.9309 & 0.8429 & 0.9843 \\
\hline & $10^{7}$ & 0.4711 & 0.8022 & 0.9308 & 0.8468 & 0.9843 \\
\hline \multirow{3}{*}{6} & $10^{5}$ & 0.6521 & 0.8693 & 0.9028 & 0.9917 & 0.9942 \\
\hline & $10^{6}$ & 0.6521 & 0.8356 & 0.9028 & 0.9919 & 0.9942 \\
\hline & $10^{7}$ & 0.6520 & 0.8260 & 0.8627 & 0.9920 & 0.9942 \\
\hline \multirow{3}{*}{7} & $10^{5}$ & 0.6635 & 0.8454 & 0.8604 & 0.9935 & 0.9693 \\
\hline & $10^{6}$ & 0.6440 & 0.8830 & 0.8073 & 0.9932 & 0.9710 \\
\hline & $10^{7}$ & 0.6440 & 0.8323 & 0.8376 & 0.9914 & 0.9691 \\
\hline \multirow{3}{*}{8} & $10^{5}$ & 0.7163 & 0.9796 & 0.9090 & 0.8775 & 0.9975 \\
\hline & $10^{6}$ & 0.7088 & 0.8478 & 0.9092 & 0.8760 & 0.9977 \\
\hline & $10^{7}$ & 0.3990 & 0.8084 & 0.8579 & 0.8850 & 0.9979 \\
\hline \multirow{3}{*}{9} & $10^{5}$ & 0.4992 & 0.8745 & 0.8678 & 0.9997 & 0.5148 \\
\hline & $10^{6}$ & 0.4012 & 0.8803 & 0.8509 & 0.9995 & 0.5148 \\
\hline & $10^{7}$ & 0.3903 & 0.8353 & 0.8345 & 0.9995 & 0.5148 \\
\hline \multirow{3}{*}{10} & $10^{3}$ & 0.0382 & 0.5973 & 0.6395 & 0.0289 & 0.0335 \\
\hline & $10^{4}$ & 0.5612 & 0.6108 & 0.6191 & 0.0282 & 0.0337 \\
\hline & $10^{5}$ & 0.4125 & 0.6088 & 0.6091 & 0.0290 & 0.0329 \\
\hline
\end{tabular}

TABle 8: The proportion of the component functions used in the active set strategy for Example 11.

\begin{tabular}{|c|c|c|c|c|c|c|}
\hline$n$ & $q$ & ASSF & SSF & SPF & TAF & ASAF \\
\hline $10^{2}$ & $10^{2}$ & 0.2741 & 0.7344 & 0.5394 & 0.8765 & 0.9228 \\
\hline $10^{2}$ & $10^{3}$ & 0.2212 & 0.7226 & 0.5087 & 0.8088 & 0.9227 \\
\hline $10^{2}$ & $10^{4}$ & 0.3030 & 0.7951 & $\mathrm{fail}^{3}$ & 0.8364 & 0.9227 \\
\hline 10 & $10^{3}$ & 0.1969 & 0.8133 & 0.5097 & 0.8009 & 0.8998 \\
\hline $10^{2}$ & $10^{3}$ & 0.2570 & 0.7975 & 0.5145 & 0.8371 & 0.8984 \\
\hline $10^{3}$ & $10^{3}$ & 0.2477 & 0.7622 & fail $^{3}$ & 0.8584 & 0.8977 \\
\hline
\end{tabular}




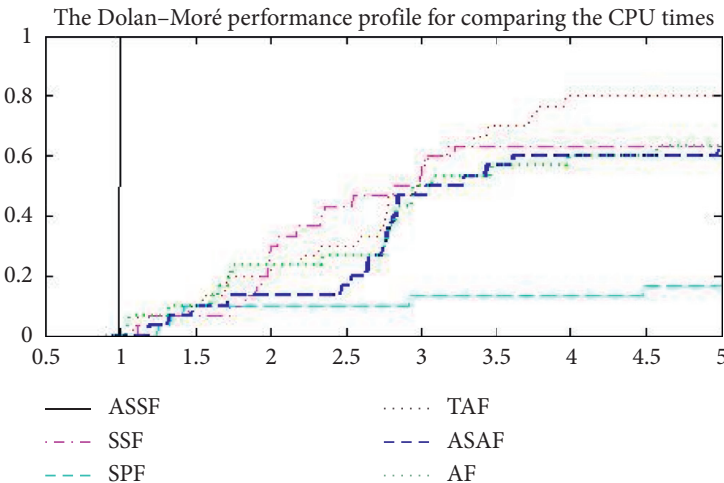

(a)

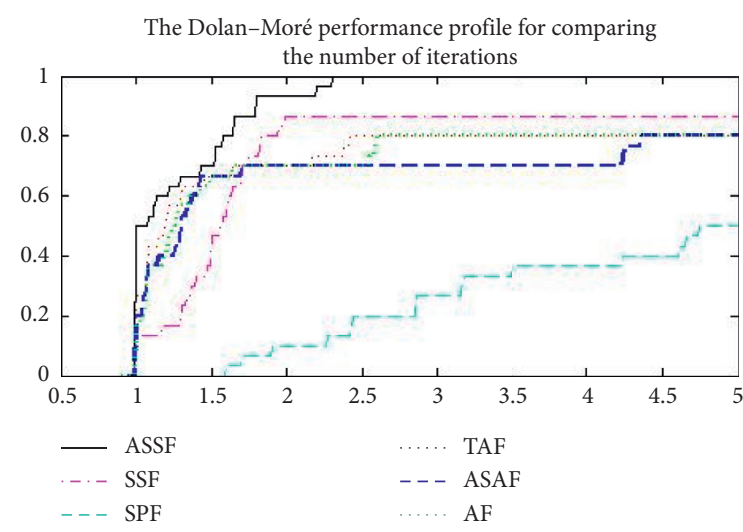

(b)

Figure 1: The Dolan-Morée performance profile of Examples 1-10.

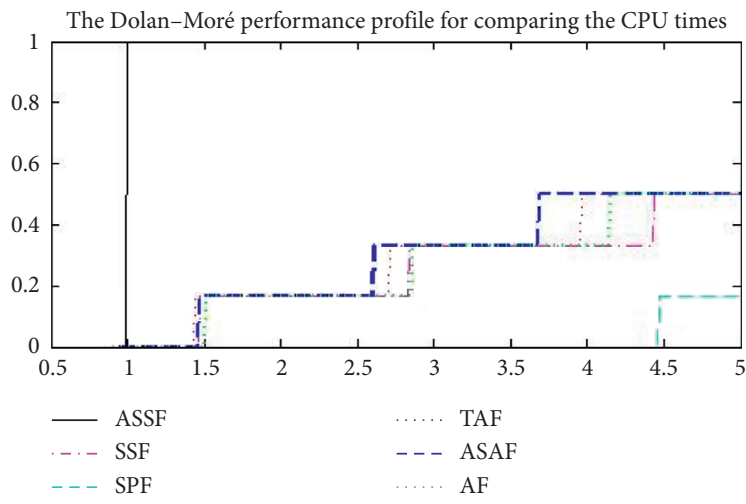

(a)

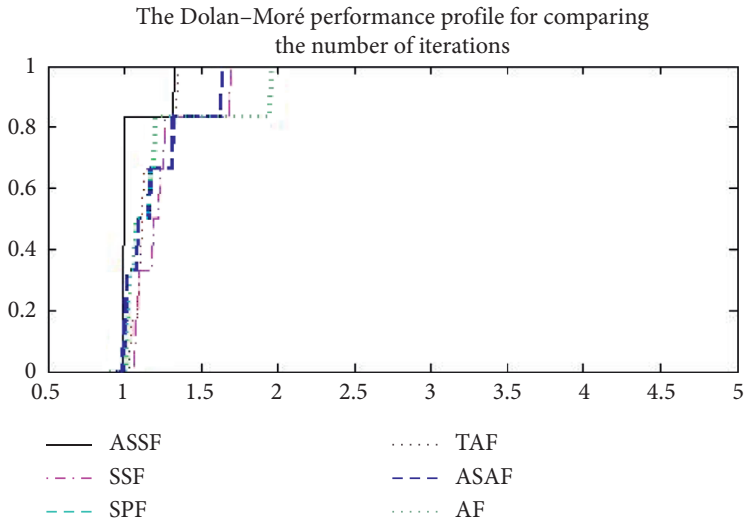

(b)

Figure 2: The Dolan-Morée performance profile of Examples 11.

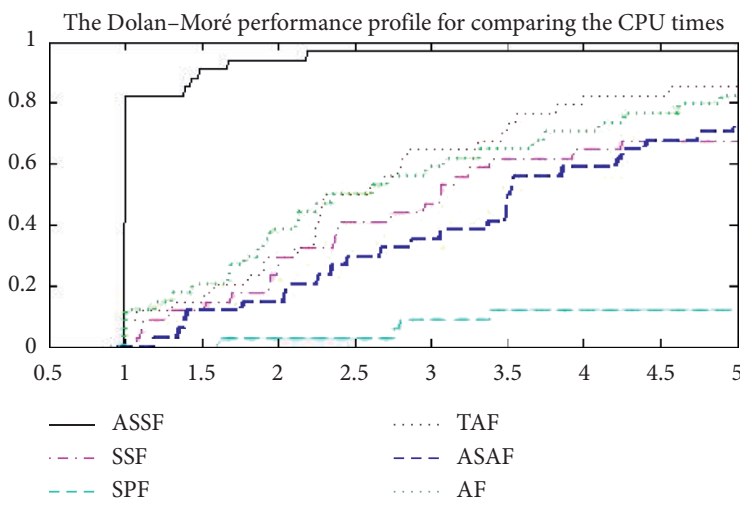

(a)

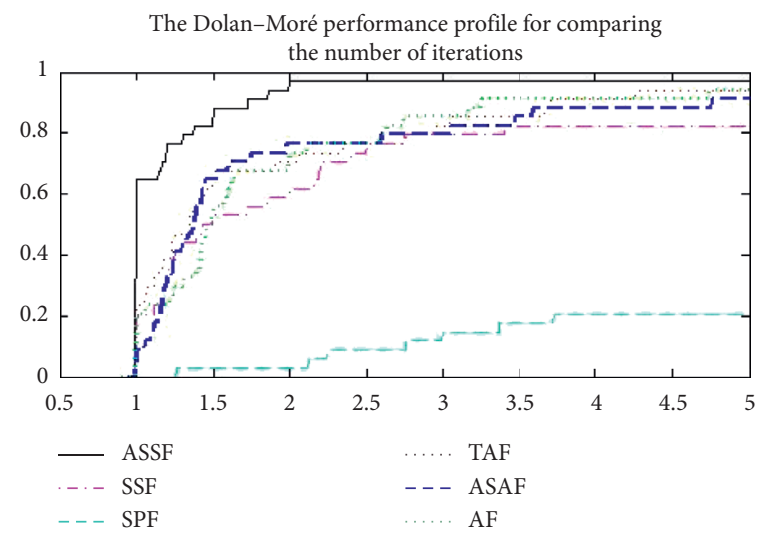

(b)

Figure 3: The Dolan-e Moré performance profile of Examples 12-25. 


\section{Conclusion}

We have proposed an active set smoothing function for the maximum function by using the plus function and an active set smoothing method with convergence analysis for solving unconstrained minimax problems. The active smoothing function can be simply implemented in the smoothing methods. Compared with the similar smoothing algorithms based on other smoothing techniques, and the algorithms in the MATLAB environment, the proposed algorithm is competitive for wide moderately sized problems and dramatically efficient for the problems with very many component functions.

\section{Appendix}

Example 1 (see [24])

$$
\begin{aligned}
F(x) & =\max _{1 \leq j \leq q} f_{j}(x), \\
f_{j}(x) & =\sin y_{j}-\left(x_{3} y_{j}^{2}+x_{2} y_{j}+x_{1}\right), \quad j=1, \ldots, \frac{q}{2}, \\
f_{j}(x) & =-f_{j-q / 2}(x), \quad j=\frac{q}{2+1}, \ldots, q, \\
y_{j} & =\frac{(j-1)}{(q / 2-1)}, \quad j=1, \ldots, \frac{q}{2},
\end{aligned}
$$

$n=3, x^{0}=(1,1,1)$.

Example 2 (see [24])

$$
\begin{aligned}
F(x) & =\max _{1 \leq j \leq q} f_{j}(x), \\
f_{j}(x) & =\left(2 y_{j}^{2}-1\right) x+y_{j}\left(1-y_{j}\right)(1-x), \quad j=1, \ldots, \frac{q}{2}, \\
f_{j}(x) & =-f_{j-q / 2}(x), \quad j=\frac{q}{2+1}, \ldots, q, \\
y_{j} & =\frac{(j-1)}{(q / 2-1)}, \quad j=1, \ldots, \frac{q}{2},
\end{aligned}
$$

$n=1, x^{0}=5$

Example 3 (see [24])

$$
\begin{aligned}
F(x) & =\max _{1 \leq j \leq q} f_{j}(x), \\
f_{j}(x) & =\sqrt{y_{j}}-\left(x_{4}-\left(x_{1} y_{j}^{2}+x_{2} y_{j}+x_{3}\right)^{2}\right), \quad j=1, \ldots, \frac{q}{2}, \\
f_{j}(x) & =-f_{j-q / 2}(x), \quad j=\frac{q}{2+1}, \ldots, q, \\
y_{j} & =0.25+0.75 \frac{(j-1)}{(q / 2-1)}, \quad j=1, \ldots, \frac{q}{2},
\end{aligned}
$$

$n=4, x^{0}=(1,1,1,1)$.
Example 4 (see [33])

$$
\begin{aligned}
& F(x)=\max _{1 \leq j \leq q} f_{j}(x), \\
& f_{j}(x)=\left(x_{1}+x_{2} y_{j}-\exp \left(y_{j}\right)\right)^{2}+\left(x_{3}+x_{4} \sin y_{j}-\cos y_{j}\right)^{2}, \\
& \quad j=1, \ldots, \frac{q}{2}, \\
& f_{j}(x)=-f_{j-q / 2}(x), \quad j=\frac{q}{2+1}, \ldots, q, \\
& y_{j}=4 \frac{(j-1)}{(q / 2-1)}, \quad j=1, \ldots, \frac{q}{2},
\end{aligned}
$$

$n=4, x^{0}=(25,5,-5,-1)$.

Example 5 (see [34])

$$
\begin{gathered}
F(x)=\max _{1 \leq j \leq q} f_{j}(x), \\
f_{j}(x)=\frac{x_{1}+x_{2} y_{j}+x_{3} y_{j}^{2}}{1+x_{4} y_{j}+x_{5} y_{j}^{2}}-\frac{\sqrt{\left(8 y_{j}-1\right)^{2}+1} \arctan \left(8 y_{j}\right)}{8 y_{j}}, \\
j=1, \ldots, \frac{q}{2},
\end{gathered}
$$

$$
\begin{aligned}
f_{j}(x) & =-f_{j-q / 2}(x), \quad j=\frac{q}{2+1}, \ldots, q, \\
y_{j} & =-1+2 \frac{(j-1)}{(q / 2-1)}, \quad j=1, \ldots, \frac{q}{2},
\end{aligned}
$$

$n=5, x^{0}=(0,-1,10,1,10)$.

\section{Example 6 (see [34])}

$$
\begin{aligned}
F(x)= & \max _{1 \leq j \leq q} f_{j}(x), \\
f_{j}(x)= & \frac{x_{3}}{x_{2}} \exp \left(-y_{j} x_{1}\right) \sin \left(y_{j} x_{2}\right)-\left(\frac{3}{20} \exp \left(-y_{j}\right)+\frac{1}{52} \exp \left(-5 y_{j}\right)\right. \\
& -\frac{1}{65} \exp \left(-2 y_{j}\right)\left(3 \sin \left(2 y_{j}\right)+11 \cos \left(2 y_{j}\right)\right), \quad j=1, \ldots, \frac{q}{2}, \\
f_{j}(x)= & -f_{j-q / 2}(x), \quad j=\frac{q}{2+1}, \ldots, q, \\
y_{j}= & \frac{10(j-1)}{(q / 2-1)}, \quad j=1, \ldots, \frac{q}{2},
\end{aligned}
$$

$n=3, x^{0}=(1,1,1)$. 
Example 7 (see [35])

$$
\begin{aligned}
F(x) & =\max _{1 \leq j \leq q} f_{j}(x), \\
f_{j}(x) & =\frac{x_{1}+x_{2} y_{j}}{1+x_{3} y_{j}+x_{4} y_{j}^{2}+x_{5} y_{j}^{3}}-\exp \left(y_{j}\right), \quad j=1, \ldots, \frac{q}{2}, \\
f_{j}(x) & =-f_{j-q / 2}(x), \quad j=\frac{q}{2+1}, \ldots, q, \\
y_{j} & =-1+2 \frac{(j-1)}{(q / 2-1)}, \quad j=1, \ldots, \frac{q}{2},
\end{aligned}
$$

$n=5, x^{0}=(0.5,0,0,0,0)$.

Example 8 (see [13])

$$
\begin{aligned}
F(x) & =\max _{1 \leq j \leq q} f_{j}(x), \\
f_{j}(x) & =x_{1} \exp \left(x_{3} y_{j}\right)+x_{2} \exp \left(x_{4} y_{j}\right)-\frac{1}{1+y_{j}}, \quad j=1, \ldots, \frac{q}{2}, \\
f_{j}(x) & =-f_{j-q / 2}(x), \quad j=\frac{q}{2+1}, \ldots, q, \\
y_{j} & =-0.5+\frac{(j-1)}{(q / 2-1)}, \quad j=1, \ldots, \frac{q}{2},
\end{aligned}
$$

$$
n=4, x^{0}=(1,1,-3,-1) .
$$

Example 9 (see [36])

$$
\begin{aligned}
F(x)= & \max _{1 \leq j \leq q} f_{j}(x), \\
f_{j}(x)= & x_{1}^{2} \exp \left(-x_{2} y_{j}\right) \cos ^{2}\left(x_{3} y_{j}+x_{4}\right)-\cos \left(y_{j}\right) \\
& +x_{2}^{2} x_{3}^{2} \exp \left(-x_{1} y_{j}\right) \sin ^{2}\left(x_{2} y_{j}\right) \\
& +\exp \left(\left(1-x_{6}\right)^{2} y_{j}+x_{5}^{2}\right), \quad j=1, \ldots, q,
\end{aligned}
$$

$$
y_{j}=\frac{10(j-1)}{(q-1)}, \quad j=1, \ldots, q,
$$

$n=6, x^{0}=(1,1,1,1,1,1)$.

Example 10 (see [36])

$$
\begin{aligned}
F(x)= & \max _{1 \leq j \leq q} f_{j}(x), \\
f_{j}(x)= & \frac{x_{3}}{x_{2}} \exp \left(-y_{j} x_{1}\right) \sin \left(y_{j} x_{2}\right)+x_{1} \exp \left(-x_{2} y_{j}\right) \cos \left(x_{3} y_{j}+x_{4}\right)+x_{5} \exp \left(-x_{6} y_{j}\right) \\
& -\left(\frac{3}{20} \exp \left(-y_{j}\right)+\frac{1}{52} \exp \left(-5 y_{j}\right)-\frac{1}{65} \exp \left(-2 y_{j}\right)\left(3 \sin \left(2 y_{j}\right)+11 \cos \left(2 y_{j}\right)\right)+\frac{1}{2} \exp \left(-y_{j}\right)\right. \\
& \left.-\exp \left(-2 y_{j}\right)+\frac{1}{2} \exp \left(-3 y_{j}\right)+\frac{3}{2} \exp \left(-\frac{3}{2} y_{j}\right) \sin \left(7 y_{j}\right)+\exp \left(-\frac{5}{2} y_{j}\right) \sin \left(5 y_{j}\right)\right), \quad j=1, \ldots, \frac{q}{2}, \\
f_{j}(x)= & -f_{j-q / 2}(x), \quad j=\frac{q}{2+1}, \ldots, q, \\
y_{j}= & \frac{10(j-1)}{(q / 2-1)}, \quad j=1, \ldots, \frac{q}{2},
\end{aligned}
$$$$
n=6, x^{0}=(2,2,7,0,-2,1) .
$$

generated randomly as normal distribution. $x^{0}=((1 / n),(1 / n), \ldots,(1 / n))$.

Example 11 (see [18])

$$
\begin{gathered}
F(x)=\max _{1 \leq j \leq q} f_{j}(x), \\
f_{j}(x)=\frac{1}{2} x^{T} A_{j} x+B_{j}^{T} x+C_{j}, \\
j=1, \ldots, q,
\end{gathered}
$$

where $A_{j} \in R^{n \times n}, j=1, \ldots, q$, is symmetric positive definite, and $B_{j} \in R^{n}, j=1, \ldots, q$, and $C_{j} \in R, j=1, \ldots, q$, are all

Example 12 (see [24])

$$
\begin{aligned}
F(x) & =\max _{1 \leq j \leq 3} f_{j}(x), \\
f_{1}(x) & =\left(x_{1}\right)^{2}+\left(x_{2}\right)^{4}, \\
f_{2}(x) & =\left(2-x_{1}\right)^{2}+\left(2-x_{2}\right)^{2}, \\
f_{3}(x) & =2 \exp \left(-x_{1}+x_{2}\right),
\end{aligned}
$$

$n=2, x^{0}=(0,0)$. 
Example 13 (see [24]).

$$
\begin{aligned}
F(x) & =\max _{1 \leq j \leq 20} f_{j}(x), \\
f_{j}(x)=x_{j}^{2}, & \\
& j=1, \ldots, 20 .
\end{aligned}
$$

$n=20, x^{0}=(0.1,0.2, \ldots, 1,-1.1,-1.2, \ldots,-2)$.

Example 14 (see [24]).

$$
\begin{aligned}
& F(x)=\max _{1 \leq j \leq 2} f_{j}(x), \\
& f_{1}(x)=\left(x_{1}-\sqrt{x_{1}^{2}+x_{2}^{2}} \cos \left(x_{1}^{2}+x_{2}^{2}\right)\right)^{2}+0.005\left(x_{1}^{2}+x_{2}^{2}\right), \\
& f_{2}(x)=\left(x_{2}-\sqrt{x_{1}^{2}+x_{2}^{2}} \sin \left(x_{1}^{2}+x_{2}^{2}\right)\right)^{2}+0.005\left(x_{1}^{2}+x_{2}^{2}\right),
\end{aligned}
$$

$n=2, x^{0}=(1.41831,-4.79462)$.

Example 15 (see [24]).

$$
\begin{aligned}
& F(x)=\max _{1 \leq j \leq 100} f_{j}(x), \\
& f_{j}(x)=x_{j}^{2}, \\
& \quad j=1, \ldots, 100,
\end{aligned}
$$

$n=100, x^{0}=(0.1,0.2, \ldots, 1,-1.1,-1.2, \ldots,-2)$.
Example 16 (see [24]).

$$
\begin{aligned}
F(x) & =\max _{1 \leq j \leq 200} f_{j}(x), \\
f_{j}(x) & =x_{j}^{2}, \\
& \quad j=1, \ldots, 200,
\end{aligned}
$$

$n=200, x^{0}=(0.1,0.2, \ldots, 1,-1.1,-1.2, \ldots,-2)$.

Example 17 (see [24]).

$$
\begin{gathered}
F(x)=\max _{1 \leq j \leq 50} f_{j}(x), \\
f_{j}(x)=x_{2(j-1)+1}^{2}+x_{2 j}^{2}, \\
j=1, \ldots, 50 \\
n=100, x^{0}=(0.02,0.04, \ldots, 1,-1.02,-1.04, \ldots,-2) .
\end{gathered}
$$

Example 18 (see [24]).

$$
\begin{aligned}
F(x)= & \max _{1 \leq j \leq 50} f_{j}(x), \\
f_{j}(x)= & x_{4(j-1)+1}^{2}+x_{4(j-1)+2}^{2}+x_{4(j-1)+3}^{2}+x_{4 j}^{2}, \\
& \quad j=1, \ldots, 50, \\
n=200, x^{0}= & (0.01,0.02, \ldots, 1,-1.01,-1.02, \ldots,-2) .
\end{aligned}
$$

Example 19. (see [33]).

$$
\begin{aligned}
F(x) & =\max _{0 \leq j \leq 30} f_{j}(x), \\
f_{j}(x) & =x_{1}+\frac{j}{(16-j) x_{2}+\min (j, 16-j) x_{3}}-y_{j}, j=1, \ldots, 15, \\
f_{j}(x) & =-f_{j-15}(x), \quad j=16, \ldots, 30, \\
y & =(0.14,0.18,0.22,0.25,0.29,0.32,0.35,0.39,0.37,0.58,0.73,0.96,1.34,2.10,4.39),
\end{aligned}
$$

$n=3, x^{0}=(1,1,1)$.

Example 20 (see [33]).

$$
\begin{aligned}
F(x) & =\max _{0 \leq j \leq 22} f_{j}(x), \\
f_{j}(x) & =\frac{x_{1}\left(u_{j}^{2}+x_{2} u_{j}\right)}{u_{j}^{2}+x_{3} u_{j}+x_{4}}-y_{j}, \quad j=1, \ldots, 11, \\
f_{j}(x) & =-f_{j-11}(x), \quad j=12, \ldots, 22, \\
y & =(0.1951,0.1947,0.1735,0.1600,0.0844,0.0627,0.0456,0.0342,0.0323,0.0235,0.0246), \\
u & =(4.0000,2.0000,1.0000,0.5000,0.2500,0.1670,0.1250,0.1000,0.0833,0.0714,0.0625),
\end{aligned}
$$


Example 21 (see [37]).

$$
\begin{aligned}
& F(x)=\max _{1 \leq j \leq 5} f_{j}(x), \\
& f_{1}(x)=\left(x_{1}-10\right)^{2}+5\left(x_{2}-12\right)^{2}+x_{3}^{4}+3\left(x_{4}-11\right)^{2}+10 x_{5}^{6}+7 x_{6}^{2}+x_{7}^{4}-4 x_{6} x_{7}-10 x_{6}-8 x_{7}, \\
& f_{2}(x)=f_{1}(x)+10\left(2 x_{1}^{2}+3 x_{2}^{4}+x_{3}+4 x_{4}^{2}+5 x_{5}-127\right), \\
& f_{3}(x)=f_{1}(x)+10\left(7 x_{1}+3 x_{2}+10 x_{3}^{2}+x_{4}-x_{5}-282\right), \\
& f_{4}(x)=f_{1}(x)+10\left(23 x_{1}+x_{2}^{2}+6 x_{6}^{2}-8 x_{7}-196\right), \\
& f_{5}(x)=f_{1}(x)+10\left(4 x_{1}^{2}+x_{2}^{2}-3 x_{1} x_{2}+2 x_{3}^{2}+5 x_{6}-11 x_{7}\right) .
\end{aligned}
$$

$n=7, x^{0}=(1,2,0,4,0,1,1)$.

Example 22 (see [37]).

$$
\begin{aligned}
F(x)= & \max _{1 \leq j \leq 9} f_{j}(x) \\
f_{1}(x)= & x_{1}^{2}+x_{2}^{2}+x_{1} x_{2}-14 x_{1}-16 x_{2}+\left(x_{3}-10\right)^{2}+4\left(x_{4}-5\right)^{2}+\left(x_{5}-3\right)^{2}+ \\
& +2\left(x_{6}-1\right)^{2}+5 x_{7}^{2}+7\left(x_{8}-11\right)^{2}+2\left(x_{9}-10\right)^{2}+\left(x_{10}-7\right)^{2}+45 \\
f_{2}(x)= & f_{1}(x)+10\left(3\left(x_{1}-2\right)^{2}+4\left(x_{2}-3\right)^{2}+2 x_{3}^{2}-7 x_{4}-120\right) \\
f_{3}(x)= & f_{1}(x)+10\left(5 x_{1}^{2}+8 x_{2}+\left(x_{3}-6\right)^{2}-2 x_{4}-40\right) \\
f_{4}(x)= & f_{1}(x)+10\left(0.5\left(x_{1}-8\right)^{2}+2\left(x_{2}-4\right)^{2}+3 x_{5}^{2}-x_{6}-30\right) \\
f_{5}(x)= & f_{1}(x)+10\left(x_{1}^{2}+2\left(x_{2}-2\right)^{2}-2 x_{1} x_{2}+14 x_{5}-6 x_{6}\right) \\
f_{6}(x)= & f_{1}(x)+10\left(4 x_{1}+5 x_{2}-3 x_{7}+9 x_{8}-105\right) \\
f_{7}(x)= & f_{1}(x)+10\left(10 x_{1}-8 x_{2}-17 x_{7}+2 x_{8}\right) \\
f_{8}(x)= & f_{1}(x)+10\left(-3 x_{1}+6 x_{2}+12\left(x_{9}-8\right)^{2}-7 x_{1} 0\right) \\
f_{9}(x)= & f_{1}(x)+10\left(-8 x_{1}+2 x_{2}+5 x_{9}-2 x_{10}-12\right)
\end{aligned}
$$

$n=10, x^{0}=(2,3,5,5,1,2,7,3,6,10)$.

Example 23 (see [37]).

$$
\begin{aligned}
F(x)= & \max _{1 \leq j \leq 18} f_{j}(x), \\
f_{1}(x)= & x_{1}^{2}+x_{2}^{2}+x_{1} x_{2}-14 x_{1}-16 x_{2}+\left(x_{3}-10\right)^{2}+4\left(x_{4}-5\right)^{2}+\left(x_{5}-3\right)^{2}+ \\
& +2\left(x_{6}-1\right)^{2}+5 x_{7}^{2}+7\left(x_{8}-11\right)^{2}+2\left(x_{9}-10\right)^{2}+\left(x_{10}-7\right)^{2}+\left(x_{11}-9\right)^{2}+ \\
& +10\left(x_{12}-1\right)^{2}+5\left(x_{13}-7\right)^{2}+4\left(x_{14}-14\right)^{2}+27\left(x_{15}-1\right)^{2}+x_{16}^{4}+\left(x_{17}-2\right)^{2}+ \\
& +13\left(x_{8}-2\right)^{2}+\left(x_{19}-3\right)^{2}+x_{20}^{2}+95, \\
f_{2}(x)= & f_{1}(x)+10\left(3\left(x_{1}-2\right)^{2}+4\left(x_{2}-3\right)^{2}+2 x_{3}^{2}-7 x_{4}-120\right), \\
f_{3}(x)= & f_{1}(x)+10\left(5 x_{1}^{2}+8 x_{2}+\left(x_{3}-6\right)^{2}-2 x_{4}-40\right), \\
f_{4}(x)= & f_{1}(x)+10\left(0.5\left(x_{1}-8\right)^{2}+2\left(x_{2}-4\right)^{2}+3 x_{5}^{2}-x_{6}-30\right), \\
f_{5}(x)= & f_{1}(x)+10\left(x_{1}^{2}+2\left(x_{2}-2\right)^{2}-2 x_{1} x_{2}+14 x_{5}-6 x_{6}\right), \\
f_{6}(x)= & f_{1}(x)+10\left(4 x_{1}+5 x_{2}-3 x_{7}+9 x_{8}-105\right),
\end{aligned}
$$




$$
\begin{aligned}
& f_{7}(x)=f_{1}(x)+10\left(10 x_{1}-8 x_{2}-17 x_{7}+2 x_{8}\right), \\
& f_{8}(x)=f_{1}(x)+10\left(-3 x_{1}+6 x_{2}+12\left(x_{9}-8\right)^{2}-7 x_{10}\right), \\
& f_{9}(x)=f_{1}(x)+10\left(-8 x_{1}+2 x_{2}+5 x_{9}-2 x_{10}-12\right), \\
& f_{10}(x)=f_{1}(x)+10\left(x_{1}+x_{2}+4 x_{11}-21 x_{12}\right), \\
& f_{11}(x)=f_{1}(x)+10\left(x_{1}^{2}+15 x_{11}-8 x_{12}-28\right), \\
& f_{12}(x)=f_{1}(x)+10\left(4 x_{1}+9 x_{2}+5 x_{13}^{2}-9 x_{14}-87\right), \\
& f_{13}(x)=f_{1}(x)+10\left(3 x_{1}+4 x_{2}+3\left(x_{13}-6\right)^{2}-14 x_{14}-10\right), \\
& f_{14}(x)=f_{1}(x)+10\left(14 x_{1}^{2}+35 x_{15}-79 x_{16}-92\right), \\
& f_{15}(x)=f_{1}(x)+10\left(15 x_{2}^{2}+11 x_{15}-61 x_{16}-54\right), \\
& f_{16}(x)=f_{1}(x)+10\left(5 x_{1}^{2}+2 x_{2}+9 x_{17}^{4}-x_{18}-68\right), \\
& f_{17}(x)=f_{1}(x)+10\left(x_{1}^{2}-x_{2}+19 x_{19}-20 x_{20}+19\right), \\
& f_{18}(x)=f_{1}(x)+10\left(7 x_{1}+5 x_{2}^{2}+x_{19}^{2}-30 x_{20}\right),
\end{aligned}
$$

$n=20, x^{0}=(2,3,5,5,1,2,7,3,6,10,2,2,6,15,1,2,1,2,1,3) . \quad$ Example 24 (see [37]).

$$
\begin{aligned}
F(x)= & \max _{1 \leq j \leq 10} f_{j}(x), \\
f_{j}(x)= & b_{j} \sum_{i=1}^{5}\left(x_{i}-a_{j i}\right)^{2}, \\
j= & 1, \ldots, 10, \\
A= & {\left[\begin{array}{ccccc}
0 & 0 & 0 & 0 & 0 \\
2 & 1 & 1 & 1 & 3 \\
1 & 2 & 1 & 1 & 2 \\
1 & 4 & 1 & 2 & 2 \\
3 & 2 & 1 & 0 & 1 \\
0 & 2 & 1 & 0 & 1 \\
1 & 1 & 1 & 1 & 1 \\
1 & 0 & 1 & 2 & 1 \\
0 & 0 & 2 & 1 & 0 \\
1 & 1 & 2 & 0 & 0
\end{array}\right], } \\
b= & {\left[\begin{array}{c}
1 \\
5 \\
10 \\
2 \\
4 \\
1.7 \\
2.5 \\
6 \\
3.5
\end{array}\right], }
\end{aligned}
$$

$n=5, x^{0}=(0,0,0,0,1)$. 
Example 25 (see [34]).

$$
\begin{aligned}
F(x)= & \max _{1 \leq j \leq 130} f_{j}(x), \\
f_{j}(x)= & t_{j}-x_{1} \exp \left(-x_{5} y_{j}\right)-x_{2} \exp \left(-x_{6}\left(y_{j}-x_{9}\right)^{2}\right)-x_{3} \exp \left(-x_{7}\left(y_{j}-x_{10}\right)^{2}\right) \\
& -x_{4} \exp \left(-x_{8}\left(y_{j}-x_{11}\right)^{2}\right), \quad j=1, \ldots, 65 \\
f_{j}(x)= & -f_{j-65}(x), \quad j=66, \ldots, 130, \\
y_{j}= & 0.1(j-1), \quad j=1, \ldots, 65 \\
t= & (1.366,1.191,1.112,1.013,0.991,0.885,0.831,0.847,0.786,0.725,0.746,0.679,0.608,0.655, \\
& 0.616,0.606,0.602,0.626,0.651,0.724,0.649,0.649,0.694,0.644,0.624,0.661,0.612,0.558, \\
& 0.533,0.495,0.500,0.423,0.395,0.375,0.372,0.391,0.396,0.405,0.428,0.429,0.523,0.562, \\
& 0.607,0.653,0.672,0.708,0.633,0.668,0.645,0.632,0.591,0.559,0.597,0.625,0.739,0.710, \\
& 0.729,0.720,0.636,0.581,0.428,0.292,0.162,0.098,0.054) .
\end{aligned}
$$

$n=11, x^{0}=(1.3,0.65,0.65,0.7,0.6,3,5,7,2,4.5,5.5)$.

Example 26 (see [38]).

$$
\begin{gathered}
F(x)=\max _{1 \leq j \leq 40} f_{j}(x), \\
f_{j}(x)=x_{j}, \quad j=1, \ldots, 20, \\
f_{j}(x)=-f_{j-20}(x), \quad j=21, \ldots, 40, \\
n=20, x^{0}=(1,2, \ldots, 10,-11,-12, \ldots,-20) .
\end{gathered}
$$

Example 27 (see [38]).

$$
\begin{array}{r}
F(x)=\max _{1 \leq j \leq 50} f_{j}(x), \\
f_{j}(x)=50 x_{j}-\sum_{i=1}^{50} x_{i}, \\
j=1, \ldots, 50,
\end{array}
$$

$n=50, x^{0}=(1-25.5, \ldots, i-25.5, \ldots, 50-25.5)$.

Example 28 (see [38]).

$$
\begin{aligned}
F(x) & =\max _{1 \leq j \leq 2} f_{j}(x), \\
f_{1}(x) & =\left(x_{1}\right)^{2}+\left(x_{2}-1\right)^{2}+x_{2}-1, \\
f_{2}(x) & =-\left(x_{1}\right)^{2}-\left(x_{2}-1\right)^{2}+x_{2}+1,
\end{aligned}
$$

$n=2, x^{0}=(-1.5,2)$.

Example 29 (see [38]).

$$
\begin{gathered}
F(x)=\max _{1 \leq j \leq 2} f_{j}(x), \\
f_{1}(x)=e^{x_{1}^{2} / 1000+\left(x_{2}-1\right)^{2}}, \\
f_{2}(x)=e^{x_{1}^{2} / 1000+\left(x_{2}+1\right)^{2}},
\end{gathered}
$$

$n=2, x^{0}=(50,0.05)$.
Example 30 (see [38]).

$$
\begin{aligned}
& F(x)=\max _{1 \leq j \leq 2} f_{j}(x), \\
& f_{1}(x)=-x_{1}-x_{2}, \\
& f_{2}(x)=-x_{1}-x_{2}+\left(x_{1}^{2}+x_{2}^{2}-1\right),
\end{aligned}
$$

$n=2, x^{0}=(-0.5,-0.5)$.

Example 31 (see [38]).

$$
\begin{aligned}
F(x) & =\max _{1 \leq j \leq 2} f_{j}(x), \\
f_{1}(x) & =-x_{1}, \\
f_{2}(x) & =-x_{1}+x_{1}^{2}+x_{2}^{2}-1,
\end{aligned}
$$

$n=2, x^{0}=(-1,-1)$.

Example 32 (see [38]).

$$
\begin{aligned}
& F(x)=\max _{1 \leq j \leq 2} f_{j}(x), \\
& f_{1}(x)=-x_{1}+2\left(x_{1}^{2}+x_{2}^{2}-1\right)+1.75\left(x_{1}^{2}+x_{2}^{2}-1\right), \\
& f_{2}(x)=-x_{1}+2\left(x_{1}^{2}+x_{2}^{2}-1\right)-1.75\left(x_{1}^{2}+x_{2}^{2}-1\right),
\end{aligned}
$$

$n=2, x^{0}=(-1,-1)$.

Example 33 (see [38]).

$$
\begin{aligned}
& F(x)=\max _{1 \leq j \leq 3} f_{j}(x), \\
& f_{1}(x)=x_{1}^{4}+x_{2}^{2}, \\
& f_{2}(x)=\left(2-x_{1}\right)^{2}+\left(2-x_{2}\right)^{2}, \\
& f_{3}(x)=e^{-x_{1}+x_{2}},
\end{aligned}
$$

$n=2, x^{0}=(2,2)$. 
Example 34 (see [38]).

$$
\begin{aligned}
& F(x)=\max _{1 \leq j \leq 3} f_{j}(x), \\
& f_{1}(x)=5 x_{1}+x_{2}, \\
& f_{2}(x)=-5 x_{1}+x_{2}, \\
& f_{3}(x)=x_{1}^{2}+x_{2}^{2}+4 x_{2},
\end{aligned}
$$

$n=2, x^{0}=(1,1)$.

Example 35 (see [38]).

$$
\begin{aligned}
& F(x)=\max _{1 \leq j \leq 3} f_{j}(x), \\
& f_{1}(x)=x_{1}^{2}+x_{2}^{2}, \\
& f_{2}(x)=x_{1}^{2}+x_{2}^{2}+10\left(-4 x_{1}-x_{2}+4\right), \\
& f_{3}(x)=x_{1}^{2}+x_{2}^{2}+10\left(-x_{1}-2 x_{2}+6\right),
\end{aligned}
$$

$n=2, x^{0}=(-1,5)$.

Example 36 (see [38]).

$$
\begin{aligned}
& F(x)=\max _{1 \leq j \leq 4} f_{j}(x), \\
& f_{1}(x)=10\left(x_{2}-x_{1}^{2}\right), \\
& f_{2}(x)=-f_{1}(x), \\
& f_{3}(x)=1-x_{1}, \\
& f_{4}(x)=-f_{3}(x),
\end{aligned}
$$

$$
n=2, x^{0}=(1.2,1) \text {. }
$$

Example 37 (see [38]). $n=4, x^{0}=(0,0,0,0)$.

$$
\begin{aligned}
& F(x)=\max _{1 \leq j \leq 4} f_{j}(x), \\
& f_{1}(x)=x_{1}^{2}+x_{2}^{2}+2 x_{3}^{2}+x_{4}^{2}-5 x_{1}-5 x_{2}-21 x_{3}+7 x_{4}, \\
& f_{2}(x)=f_{1}(x)+10\left(x_{1}^{2}+x_{2}^{2}+x_{3}^{2}+x_{4}^{2}+x_{1}-x_{2}+x_{3}-x_{4}-8\right), \\
& f_{3}(x)=f_{1}(x)+10\left(x_{1}^{2}+2 x_{2}^{2}+x_{3}^{2}+2 x_{4}^{2}-x_{1}-x_{4}-10\right), \\
& f_{4}(x)=f_{1}(x)+10\left(x_{1}^{2}+x_{2}^{2}+x_{3}^{2}+2 x_{1}-x_{2}-x_{4}-5\right),
\end{aligned}
$$

Example 38 (see [38]).

$$
\begin{aligned}
F(x) & =\max _{1 \leq j \leq 2} f_{j}(x), \\
f_{1}(x) & =f(x+2 a), \\
f_{2}(x) & =f(x-2 a),
\end{aligned}
$$

where

$$
\begin{aligned}
f(x) & =e^{\left(0.0001 x_{1}\right)^{2}+x_{2}^{2}+x_{3}^{2}+2 x_{4}^{2}+x_{5}^{2}+x_{6}^{2}+\cdots+x_{10}^{2}}, \\
a & =(0,1,0,0,0,0,0,0,0,0),
\end{aligned}
$$

$n=10, x^{0}=(100,0.1, \ldots, 0.1)$.

Example 39 (see [38]).

$$
\begin{gathered}
F(x)=\max _{1 \leq j \leq 10} f_{j}(x), \\
f_{j}(x)=\sum_{i=1}^{11} \frac{1}{i-j+1} e^{\left[\left(x_{i}-\sin (j-1+2(i-1))\right)^{2}\right]}, \\
n=11, x^{0}=(1, \ldots, 1) .
\end{gathered}
$$

Example 40 (see [38]).

$n=2, x^{0}=(3,1)$.

$$
F(x)=\max _{1 \leq j \leq 3} f_{j}(x),
$$$$
f_{1}(x)=\frac{1}{2}\left(x_{1}+\frac{10 x_{1}}{\left(x_{1}+0.1\right)}+2 x_{2}^{2}\right)
$$$$
f_{2}(x)=\frac{1}{2}\left(-x_{1}+\frac{10 x_{1}}{\left(x_{1}+0.1\right)}+2 x_{2}^{2}\right) \text {, }
$$$$
f_{3}(x)=\frac{1}{2}\left(x_{1}-\frac{10 x_{1}}{\left(x_{1}+0.1\right)}-2 x_{2}^{2}\right),
$$

$n=2, x^{0}=(3,1)$.

Example 41 (see [38]).

$$
\begin{aligned}
F(x) & =\max _{1 \leq j \leq 6} f_{j}(x), \\
f_{1}(x) & =x_{1}^{2}+x_{2}^{2}+x_{1} x_{2}, \\
f_{2}(x) & =-f_{1}(x) \\
f_{3}(x) & =\sin \left(x_{1}\right) \\
f_{4}(x) & =-f_{3}(x) \\
f_{5}(x) & =\cos \left(x_{2}\right) \\
f_{6}(x) & =-f_{5}(x)
\end{aligned}
$$


Example 42 (see [38]).

$$
\begin{aligned}
& F(x)=\max _{1 \leq j \leq 2} f_{j}(x), \\
& f_{1}(x)=50\left(x_{1}+x_{2}^{4}-1\right)^{2}+3 x_{1}^{2}, \\
& f_{2}(x)=50\left(x_{1}-x_{2}^{4}+1\right)^{2}+3 x_{1}^{2},
\end{aligned}
$$

$n=2, x^{0}=(0.1,0.1)$.

Example 43 (see [39]).

$$
\begin{aligned}
& F(x)=\max _{1 \leq j \leq 6} f_{j}(x), \\
& f_{1}(x)=x_{1}^{2}+x_{2}^{2}+x_{3}^{2}-1, \\
& f_{2}(x)=x_{1}^{2}+x_{2}^{2}+\left(x_{3}-2\right)^{2}, \\
& f_{3}(x)=x_{1}+x_{2}+x_{3}-1, \\
& f_{4}(x)=x_{1}+x_{2}-x_{3}+1, \\
& f_{5}(x)=2 x_{1}^{3}+6 x_{2}^{2}+2\left(5 x_{3}-x_{1}+1\right)^{2}, \\
& f_{6}(x)=x_{1}^{2}-9 x_{3},
\end{aligned}
$$

$n=3, x^{0}=(1,1,1)$.

$$
\begin{aligned}
F(x)= & \max _{1 \leq j \leq 4} f_{j}(x), \\
f_{1}(x)= & \left(x_{1}-\left(x_{4}+1\right)^{4}\right)^{2}+\left(x_{2}-\left(x_{1}-\left(x_{4}+1\right)^{4}\right)^{4}\right)^{2}+2 x_{3}^{2}+x_{4}^{2}- \\
& -5\left(x_{1}-\left(x_{4}+1\right)^{4}\right)-5\left(x_{2}-\left(x_{1}-\left(x_{4}+1\right)^{4}\right)^{4}\right)-21 x_{3}+7 x_{4}, \\
f_{2}(x)= & f_{1}(x)+10\left(\left(x_{1}-\left(x_{4}+1\right)^{4}\right)^{2}+\left(x_{2}-\left(x_{1}-\left(x_{4}+1\right)^{4}\right)^{4}\right)^{2}+x_{3}^{2}+x_{4}^{2}+\right. \\
& \left.+\left(x_{1}-\left(x_{4}+1\right)^{4}\right)-\left(x_{2}-\left(x_{1}-\left(x_{4}+1\right)^{4}\right)^{4}\right)+x_{3}-x_{4}-8\right), \\
f_{3}(x)= & f_{1}(x)+10\left(\left(x_{1}-\left(x_{4}+1\right)^{4}\right)^{2}+2\left(x_{2}-\left(x_{1}-\left(x_{4}+1\right)^{4}\right)^{4}\right)^{2}+x_{3}^{2}+2 x_{4}^{2}-\right. \\
& \left.-\left(x_{1}-\left(x_{4}+1\right)^{4}\right)-x_{4}-10\right)^{-} \\
f_{4}(x)= & f_{1}(x)+10\left(\left(x_{1}-\left(x_{4}+1\right)^{4}\right)^{2}+\left(x_{2}-\left(x_{1}-\left(x_{4}+1\right)^{4}\right)^{4}\right)^{2}+x_{3}^{2}+\right. \\
& \left.+2\left(x_{1}-\left(x_{4}+1\right)^{4}\right)-\left(x_{2}-\left(x_{1}-\left(x_{4}+1\right)^{4}\right)^{4}\right)-x_{4}-5\right),
\end{aligned}
$$


$n=4, x^{0}=(0,0,0,0)$.

Example 45 (see [41]).

$$
\begin{aligned}
F(x)= & \max _{1 \leq j \leq 82} f_{j}(x), \\
f_{j}(x)= & \left(\frac{\left(x_{1}+\left(1+x_{2}\right) \cos \vartheta_{j}\right)^{2}+\left(\left(1-x_{2}\right) \sin \vartheta_{j}\right)^{2}}{\left(x_{3}+\left(1+x_{4}\right) \cos \vartheta_{j}\right)^{2}+\left(\left(1-x_{4}\right) \sin \vartheta_{j}\right)^{2}}\right)^{1 / 2} \\
& \cdot\left(\frac{\left(x_{5}+\left(1+x_{6}\right) \cos \vartheta_{j}\right)^{2}+\left(\left(1-x_{6}\right) \sin \vartheta_{j}\right)^{2}}{\left(x_{7}+\left(1+x_{8}\right) \cos \vartheta_{j}\right)^{2}+\left(\left(1-x_{8}\right) \sin \vartheta_{j}\right)^{2}}\right)^{1 / 2} x_{9}-y_{j}, \\
y_{j}= & 1-2 t_{j}, \quad 1 \leq j \leq 41, \\
y_{j}= & -1+2 t_{j}, \quad 42 \leq j \leq 82, \\
\vartheta_{j}= & \pi t_{j}, \quad 1 \leq j \leq 41, \\
t_{j}= & 0.01(j-1), \quad 1 \leq j \leq 6, \\
t_{j}= & 0.07+0.03(j-7), \quad 7 \leq j \leq 20, \\
t_{21}= & 0.50, \\
t_{j}= & 0.54+0.03(j-22), \quad 22 \leq j \leq 35, \\
t_{j}= & 0.95+0.01(j-36), \quad 36 \leq j \leq 41, \\
t_{j}= & t_{j-41}, \quad 42 \leq j \leq 82,
\end{aligned}
$$

$n=9, x^{0}=(0,1,0,-0.15,0,-0.68,0,-0.72,0.37)$.

\section{Data Availability}

The data used to support the findings of this study are available from the corresponding author upon request.

\section{Conflicts of Interest}

The authors declare that there are no conflicts of interest regarding the publication of this paper.

\section{Acknowledgments}

This study was supported by the National Natural Science Foundation of China (no. 11701350).

\section{References}

[1] J. F. Sturm and S. Zhang, "A dual and interior-point approach to solve convex min-max problems," in Minimax and Applications, Nonconvex Optimization and Its Application, vol. 4, pp. 69-78, D. Z. Du and P. M. Pardalos, Ed, Kluwer Academic, Dordrecht, Netherlands, 1995.

[2] D. Applegate, W. Cook, S. Dash, and A. Rohe, "Solution of a min-max vehicle routing problem," INFORMS Journal on Computing, vol. 14, no. 2, pp. 132-143, 2002.
[3] E. M. Arkin, R. Hassin, and A. Levin, "Approximations for minimum and min-max vehicle routing problems," Journal of Algorithms, vol. 59, no. 1, pp. 1-18, 2006.

[4] N. V. Banichuk, "Minimax approach to structural optimization problems," Journal of Optimization Theory and Applications, vol. 20, no. 1, pp. 111-127, 1976.

[5] S. E. Sussman-Fort, "Approximate direct-search minimax circuit optimization," International Journal Numerial Methods in Engineering, vol. 28, no. 2, pp. 359-368, 1989.

[6] R. T. Pockafellar, "Computational schemes for large-scale problems in extended linear-quadratic programming," Mathematical Programming, vol. 48, no. 1-3, pp. 447-474, 1990.

[7] R. T. Rockafellar, "Linear-quadratic programming and optimal control," SIAM Journal on Control and Optimization, vol. 25, no. 3, pp. 781-814, 1987.

[8] J. A. Chatelon, D. W. Hearn, and T. J. Lowe, "A subgradient algorithm for certain minimax and minisum problems," Mathematical Programming, vol. 15, no. 1, pp. 130-145, 1978.

[9] M. Gaudioso and M. F. Monaco, "A bundle type approach to the unconstrained minimization of convex nonsmooth functions," Mathematical Programming, vol. 23, no. 1, pp. 216-226, 1982.

[10] A. Frangioni, "Generalized bundle methods," SIAM Journal on Optimization, vol. 13, no. 1, pp. 117-156, 2002.

[11] R. Reemtsen, "A cutting plane method for solving minimax problems in the complex plane," Numerical Algorithms, vol. 2, no. 3, pp. 409-436, 1992.

[12] R. S. Womersley and R. Fletcher, "An algorithm for composite nonsmooth optimization problems," Journal of Optimization Theory and Applications, vol. 48, no. 3, pp. 493-523, 1986.

[13] J. L. Zhou and A. L. Tits, "An SQP algorithm for finely discretized continuous minimax problems and other minimax problems with many objective functions," SIAM Journal on Optimization, vol. 6, no. 2, pp. 461-487, 1996.

[14] W. Murray and M. L. Overton, "A projected Lagrangian algorithm for nonlinear minimax optimization," SIAM Journal on Scientific and Statistical Computing, vol. 1, no. 3, pp. 345-370, 1980.

[15] J. F. Sturm and S. Zhang, "A dual and interior point approach to solve convex min-max problems," Nonconvex Optimization and Its Applications, pp. 69-78, 1995.

[16] L. Luksan and J. Vlcek, "Primal interior-point method for large sparse minimax optimization," Kybernetika -Praha-, vol. 45, no. 5, pp. 841-864, 2009.

[17] E. Obasanjo, G. Tzallas-Regas, and B. Rustem, "An interiorpoint algorithm for nonlinear minimax problems," Journal of Optimization Theory and Applications, vol. 144, no. 2, pp. 291-318, 2010.

[18] Y. Feng, L. Hongwei, Z. Shuisheng, and L. Sanyang, "A smoothing trust-region Newton-CG method for minimax problem," Applied Mathematics and Computation, vol. 199, no. 2, pp. 581-589, 2008.

[19] I. Zang, "A smoothing-out technique for min-max optimization," Mathematical Programming, vol. 19, no. 1, pp. 61-77, 1980.

[20] D. Q. Mayne and E. Polak, "Nondifferential optimization via adaptive smoothing," Journal of Optimization Theory and Applications, vol. 43, no. 4, pp. 601-613, 1984.

[21] R. A. Polyak, "Smooth optimization methods for minimax problems," SIAM Journal on Control and Optimization, vol. 26, no. 6, pp. 1274-1286, 1988. 
[22] L. Xingsi, "An entropy-based aggregate method for minimax optimization," Engineering Optimization, vol. 18, no. 4, pp. 277-285, 1992.

[23] S. Xu, "Smoothing method for minimax problems," Computational Optimization and Applications, vol. 20, no. 3, pp. 267-279, 2001.

[24] E. Polak, J. O. Royset, and R. S. Womersley, "Algorithms with adaptive smoothing for finite minimax problems," Journal of Optimization Theory and Applications, vol. 119, no. 3, pp. 459-484, 2003.

[25] E. Polak, R. S. Womersley, and H. X. Yin, "An algorithm based on active sets and smoothing for discretized semi-infinite minimax problems," Journal of Optimization Theory and Applications, vol. 138, no. 2, pp. 311-328, 2008.

[26] Y. Xiao and B. Yu, "A truncated aggregate smoothing Newton method for minimax problems," Applied Mathematics and Computation, vol. 216, no. 6, pp. 1868-1879, 2010.

[27] X. S. Li, "An aggregate function method for nonlinear programming," Science in China Series A-Mathematics, vol. 34, pp. 1467-1473, 1991.

[28] L. Dong and B. Yu, "A spline smoothing Newton method for finite minimax problems," Journal of Engineering Mathematics, vol. 93, no. 1, pp. 145-158, 2014.

[29] A. Fischer, "Solution of monotone complementarity problems with locally Lipschitzian functions," Mathematical Programming, vol. 76, pp. 513-532, 1997.

[30] L. Qi, D. Sun, and G. Zhou, "A new look at smoothing Newton methods for nonlinear complementarity problems and box constrained variational inequalities," Mathematical Programming, vol. 87, no. 1, pp. 1-35, 2000.

[31] L. Qi, "Convergenece analysis of some algorithms for solving nonsmooth equations," Mathematics of Operations Research, vol. 18, no. 1, pp. 227-244, 1993.

[32] E. D. Dolan and J. J. Moré, "Benchmarking optimization software with performance profiles," Mathematical Programming, vol. 91, no. 2, pp. 201-213, 2002.

[33] G. A. Watson, "The minimax solution of an overdetermined system of non-linear equations," IMA Journal of Applied Mathematics, vol. 23, no. 2, pp. 167-180, 1979.

[34] J. R. Popovic, J. W. Bandler, and C. Charalambous, "General programmes for least $p$-th and near minimax approximation," International Journal on Systems Science, vol. 5, no. 10, pp. 907-932, 1974

[35] J. Hald and K. Madsen, "Combined LP and quasi-Newton methods for minimax optimization," Mathematical Programming, vol. 20, no. 1, pp. 42-62, 1981.

[36] Y. Xiao and B. Yu, Truncated Aggregate Smoothing Algorithms, Dalian University of Technology, Dalian, China, 2010.

[37] J. Asaadi, "A computational comparison of some non-linear programs," Mathematical Programming, vol. 4, no. 1, pp. 144-154, 1973.

[38] G. D. Pillo, L. Grippo, and S. Lucidi, "A smooth method for the finite minimax problem," Mathematical Programming, vol. 60, no. 1-3, pp. 187-214, 1993.

[39] R. A. El-Attar, M. Vidyasagar, and S. R. K. Dutta, "An algorithm for $l_{1}$-norm minimization with application to nonlinear $l_{1}$-approximation," SIAM Journal on Numerical Analysis, vol. 16, no. 1, pp. 70-86, 1979.

[40] E. Polak, D. Q. Mayne, and J. E. Higgins, "Superlinearly convergent algorithm for min-max problems," Journal of Optimization Theory and Applications, vol. 69, no. 3, pp. 407-439, 1991.
[41] C. Charalambous, "Acceleration of the least $p$ th algorithm for minimax optimization with engineering applications," Mathematical Programming, vol. 17, no. 1, pp. 270-297, 1979. 